\title{
Evaluation Au Laboratoire De L'effet Larvicide Du Baume De Cajou Et De Trois Huiles Végétales À Base Des Plantes De Tephrosia Purpurea, Ricinus Communis Et Thevetia Neriifolia Pour Contrôler Les Populations De Plutella Xylostella L. 1758 (Lepidoptere, Plutellidae)
}

\section{Fernand A. Sotondji,}

Faculté des Sciences et Techniques, Université d’Abomey-Calavi, Bénin

\section{Cocou Angelo Djihinto,}

Maître de Recherche (CAMES) à l'Institut National des Recherches

Agricoles du Bénin

\section{Douro Kpindou O. Kobi,}

Chargé de Recherche (CAMES) Chercheur entomologiste à IITA, Bénin

\section{Elie Dannon,}

Enseignant Chercheur en Entomologie Agricole, Université Nationale des

Sciences, Technologies, Ingénieries, et de Mathématiques d'Abomey

(UNSTIM), Bénin

\section{Gildas Zodome,}

Faculté des lettres Arts et Sciences Humaines, Université d’Abomey-Calavi, Bénin

\section{Euloge Adjou,}

Enseignant- Chercheur en Technologie Alimentaire à l'Ecole Polytechnique d'Abomey-Calavi, Bénin

\section{Daniel C. Chougourou,}

Enseignant Chercheur au Département Génie de l'Environnement à l'Ecole Polytechnique d'Abomey-Calavi (EPAC), Bénin

\section{Mohamed M. Soumanou,}

Professeur titulaire en Biochimie à l'Ecole Polytechnique d'Abomey-Calavi, Bénin

\section{Résumé}

Cette étude a évalué l'effet larvicide du baume de cajou extrait à froid et de trois huiles végétales à base des plantes de Tephrosia purpurea, Ricinus communis et Thevetia neriifolia, dans les conditions d'élevage au laboratoire 
de Plutella xylostella L. 1758 (Lepidoptera, Plutellidae) insecte ravageur du chou au Bénin. L'efficacité des biopesticides étudiés a été comparée à celle d'un insecticide de synthèse (Lambda cyalothrine) et à celle d'un pesticide botanique de référence (Topbio). Des tests ont été réalisés sur les larves des stades 2 et 3 de P. xylostella. Deux expérimentations ont été réalisées dans cette étude. Dans la première expérimentation, les produits ont été testés sur le stade L3 de P. xylostella, avec les doses de 100\%, 75\%, 50\%, 25\% et 10\% de chacun des produits végétaux et de Lambda Cyhalothrine. A la deuxième expérimentation, les mêmes produits végétaux ont été testées sur le stade L2 du même insecte avec les doses de $50 \%, 25 \%, 10 \%$, $5 \%$, 3\%, $2 \%$ et $1 \%$ comparées au témoin de référence Lambda cyalothrine aux doses de $10 \%, 5 \%$, $3 \%, 2 \%, 1 \%, 0,5 \%$. Les mortalités ont été observées toutes les 24 heures jusqu'au neuvième jour après exposition. Le dispositif utilisé pour les tests biologiques est un bloc complètement randomisé. La formule d'Abbott a été utilisée pour corriger les taux de mortalité au niveau des insectes traités. Les variables mesurées sont la mortalité des larves, la formation des chrysalides et l'émergence des adultes. Ces variables sont soumises à une analyse de variance avec le logiciel XLSTAT version 2019. Les DL 50 pour cinq jours ont été déterminées suivant le modèle de regression de Cox de détermination de la dose correspondante à une proportion. Les résultats ont montré que les huiles végétales et le baume de cajou sont les biopesticides prometteurs pour le contrôle des populations de P. xylostella. Pour la première expérimentation, les taux de mortalité des larves L3 de $P$. xylostella n'étaient pas significativement différents entre les doses d'application de 25\% de Topbio et d'huile de $T$. purpurea. Des mortalités de 100\% ont été enregistrées avec les doses de $100 \%$, $75 \%, 50 \%$ et $25 \%$ de tous les produits utilisés sur le stade L2 et L3. Lors de la deuxième expérimentation, $100 \%$ de mortalité ont été enregistrées à une dose d'application de $10 \%$ respectivement pour le Topbio, l'huile de $T$. purpurea, le CNSL, et l'huile de R. communis. Aucune différence significative n'a été enregistrée au seuil de 5\% entre les doses de 10\% d'huile de $T$. purpurea et 3\% du témoin de référence Lambda cyalothrine. Les témoins ont enregistré le plus fort taux de chrysalides et d'adultes. Ces deux paramètres ont été fonction de la dose. Pour tous les produits utilisés, quel que soit le stade larvaire de $P$. xylostella, la réponse effet/dose a été significative. Le baume de cajou et les trois huiles végétales se positionnent comme une alternative aux pesticides de synthèses pouvant être utilisé en culture maraîchère.

Mots-clés : Plutella Xylostella, Huile Végétale, Doses Léthale, Larve, Expérimentation Au Laboratoire, Lutte Biologique. 


\section{Evaluation Au Laboratoire De L'effet Larvicide Du Baume De Cajou Et De Trois Huiles Végétales À Base Des Plantes De Tephrosia Purpurea, Ricinus Communis Et Thevetia Neriifolia Pour Contrôler Les Populations De Plutella Xylostella L. 1758 (Lepidoptere, Plutellidae)}

\section{Fernand A. Sotondji,}

Faculté des Sciences et Techniques, Université d'Abomey-Calavi, Bénin

\section{Cocou Angelo Djihinto,}

Maître de Recherche (CAMES) à l'Institut National des Recherches

Agricoles du Bénin

\section{Douro Kpindou O. Kobi,}

Chargé de Recherche (CAMES) Chercheur entomologiste à IITA, Bénin

\section{Elie Dannon,}

Enseignant Chercheur en Entomologie Agricole, Université Nationale des

Sciences, Technologies, Ingénieries, et de Mathématiques d'Abomey

(UNSTIM), Bénin

\section{Gildas Zodome,}

Faculté des lettres Arts et Sciences Humaines, Université d’Abomey-Calavi, Bénin

\section{Euloge Adjou,}

Enseignant- Chercheur en Technologie Alimentaire à l'Ecole Polytechnique d'Abomey-Calavi, Bénin

\section{Daniel C. Chougourou,}

Enseignant Chercheur au Département Génie de l'Environnement à l'Ecole Polytechnique d'Abomey-Calavi (EPAC), Bénin

\section{Mohamed M. Soumanou,}

Professeur titulaire en Biochimie à l'Ecole Polytechnique d'Abomey-Calavi, Bénin

\section{Abstract}

This study aims to evaluate the larvicidal effect of three vegetable oils: Tephrosia purpurea, Ricinus communis, Thevetia neriifolia, and cashew balm in the management of Plutella xylostella L. 1758 (Lepidoptere, plutellidae) pest insects of cabbage in the laboratory. The efficacy of the extracts has been compared to that of a synthetic insecticide (Lambda cyalothrin) and that of a 
botanical pesticide (Topbio). Larval tests were carried out on larvae of stages 2 and 3. Two experiments were set up in this study. In the first experiment, the products were tested on the L3 stage of P. xylostella, with the doses of $100 \%, 75 \%, 50 \%, 25 \%$ and $10 \%$ while in the second, the same products were tested on the L2 stage of the same insect with the doses of $50 \%, 25 \%, 10 \%$, $5 \%, 3 \%, 2 \%$ and $1 \%$ compared to the reference control Lambda cyalothrin at the doses of $10 \%, 5 \%, 3 \%, 2 \%, 1 \%, 0.5 \%$. The mortalities were read 24 hours after exposure until the ninth day. The device used for biological tests is a completely randomized block. Abbott's formula has been used to correct mortality rates for treated insects. The variables measured are the mortality rate, the formation of pupae and the emergence of adults. These variables are subjected to an analysis of variance with the XLSTAT software version 2019. The LD 50 for eight days were determined according to the Cox regression method of determining the dose corresponding to a proportion. The results showed that vegetable oils and cashew balm are the promising species in the management of dose-dependent $P$. xylostella. For the first experiment, the mortality rates of L3 larvae of $P$. xylostella were not significantly different between the 25\% application doses of Topbio and T. purpurea. Mortalities ranging from $100 \%$ were recorded with the doses of $100 \%, 75 \%, 50 \%$ and $25 \%$ of all the products used on the L2 and L3 stages. During the second experiment, $100 \%$ mortality was recorded at an application dose of $10 \%$ respectively for Topbio, $T$. purpurea, CNSL, and R. communis. No significant difference was recorded at the 5\% threshold between the $10 \%$ doses of $T$. purpurea and 3\% of the reference control Lambda cyalothrin. Witnesses recorded the highest rate of pupae and adults. These two parameters were a function of the dose. For all the products used, whatever the larval stage of $P$. xylostella, the effect / dose response was significant. Cashew balm and the three vegetable oils are positioned as an alternative to synthetic pesticides that can be used in market gardening.

Keywords: Plutella Xylostella, Vegetable Oil, Lethal Doses, Larva, Laboratory Experimentation, Biological Control.

\section{Introduction}

Au moment où la population urbaine mondiale a dépassé pour la première fois la population rurale, l'approvisionnement en denrées disponibles et saines est l'un des principaux enjeux pour les décennies à venir (Argenti 2010). A l'échelle mondiale, les cultures légumières jouent un rôle primordial dans les programmes de sécurité alimentaire et nutritionnelle (James et al. 2010). La culture des Brassicacées représente une importante ressource alimentaire, avec plus de 70 millions de tonnes produites dans le monde (FAOSTAT, 2013). Cette famille comprend 350 genres dont 3500 espèces cultivées et sauvages 
(Warwick et al. 2003) dont le chou représente l'une des plus importantes. L'Afrique ne représente que 5,8\% de la production mondiale (FAOSTAT 2013). Au Bénin, James et al., (2005) ont montré que sur 11 cultures maraîchères principalement cultivées au Sud du pays, le chou, Brassica oleracea L. (Capparales: Brassicaceae), est l'une des cultures les plus attaquées par un complexe de ravageurs dont Plutella xylostella L. (Lepidoptera : Plutellidea). La pression exercée par les bioagresseurs constitue un frein majeur à l'augmentation de la productivité et à l'amélioration de la qualité de la production de chou. La teigne du chou, $P$. xylostella (L.) (Lepidoptera : Plutellidae), représente parmi les lépidoptères, le principal ravageur du chou dans de nombreuses régions du monde. Elle provoque à elle seule des pertes pouvant atteindre plus de 90\% (Verkerk and Wright, 1996; Sarfraz et al. 2005 ; Mondédji, 2010). C’est une espèce oligophage qui se nourrit exclusivement des plantes de la famille des Brassicacées (ex : Crucifères). Elle est attirée par les composés soufrés appelés glucosinolates, caractéristiques de cette famille végétale. Elle vit essentiellement sur les choux et les autres Brassicacées cultivées (moutarde, colza, navet, etc.), mais on la trouve aussi sur les espèces sauvages (bourse à pasteur, cardamine, ravenelle, etc.) qui peuvent servir de réservoir durant les périodes où les cultures ne sont pas disponibles (Muhamad et al. 1994). Ces composés chimiques constituent des phagostimulants pour les chenilles et des stimulants de l'oviposition chez les femelles (Gupta \& Thornsteinson 1998). Face à ces ravageurs, l'emploi des insecticides de synthèse constitue la principale méthode de lutte à laquelle les agriculteurs font recours. Cependant, leur utilisation a montré certaines limites : impact sur la biodiversité, dont l'élimination des ennemis naturels (Furlong et al 2012) ; impact sur la santé des utilisateurs et des consommateurs (résidus de pesticides), augmentation des coûts de production (Chilcutt \& Tabashnik 1997) ; apparition de souches résistantes (Hooks \& Johnson 2003 ; Macharia et al. 2005 ; Sarfraz \& Keddie 2005 ; Shelton et al. 2007 ; Huang et al. 2010). En effet, les maraîchers béninois utilisent pour la protection de chou, 46 litres de pesticides chimiques concentrés en 19 applications par hectare (James et al., 2005). Une étude diagnostique des problèmes phytosanitaires réalisée au Bénin sur la protection des légumes au Sud du Bénin a montré que le chou est l'une des cultures exigeant une gamme variée et une forte utilisation de pesticides chimique dangereux dans l'agroécosystème urbain et périurbain (James et al., 2005). Malheureusement, la résistance de $P$. xylostella aux pesticides est largement répandue (Guilloux, 2000). Plutella xylostella a montré une résistance à presque tous les insecticides agricoles et même au bio-pesticide à base de la toxine de Bacillus thuringiensis (Bt) (Tabashnik et al., 1990 ; Liu et al., 1995). Ce fait, associé aux problématiques de la lutte chimique, pousse à penser que des méthodes alternatives de luttes efficaces contre ces ravageurs, sont plus que nécessaires. 
C'est dans cette optique que l'on a effectué cette étude visant à répondre à cette situation plus ou moins critique. Pour juguler la résistance développée par ces insectes, différentes méthodes alternatives ont été mises en place. Au nombre de celles-ci figure l'utilisation de plus en plus croissante d'extraits de plantes (Glitho et al., 2008). L'une des mesures alternatives dans la gestion de la résistance est l'usage des outils et méthodes de lutte non chimiques pour autant qu'ils contribuent efficacement à lutter contre la teigne du chou. D'une façon générale, les extraits de plantes pesticides sont moins dangereux que les pesticides de synthèse (Wainwright et al., 2013), même si certains extraits de plantes comme la nicotine peuvent être toxiques à certaines doses sur les organismes vivants (Slotkin et al., 2016). La décomposition assez rapide et la faible action polluante sont des avantages des extraits de plantes (Wainwright et al., 2013). Dans certaines conditions, les extraits de plantes peuvent avoir une efficacité comparable à celle des insecticides classiques. Si cette dernière efficacité n’est pas complète, elle peut néanmoins permettre de maintenir la population des ravageurs en dessous du seuil de nuisibilité et réduire l'usage des pesticides de synthèse utilisés sur les légumes. C'est pourquoi nous nous sommes intéressés aux extraits de l'huile végétale des graines de trois espèces végétales à savoir: Tephrosia purpurea, Ricinus communis, thevetia neriifolia et le baume de cajou, connus pour leurs propriétés biocides ; dans la gestion de $P$. xylostella sur le chou. Cette étude a permis d'évaluer les propriétés larvicides du Cahew Nut Shell Liquide (CNSL) extraction à froid, de $T$. purpurea, de $R$. communis, de T. neriifolia, comparé au Topbio et Lambda cyalothrine sur les populations de $P$. xylostella en culture maraîchère au Bénin. Pour développer une stratégie efficace, des études de doses-réponses ont été réalisées. Différentes doses de chaque pesticide botanique et les deux produits témoins commercialisés ont été testées sur les larves de $P$. xylostella, les larves ayant survécu ont été suivies jusqu'à l'obtention des chrysalides puis des adultes.

\section{Matériel Et Méthodes}

\subsection{Milieu D'étude}

Les expérimentations ont été conduites dans les laboratoires des biopesticides de l'IITA (Bénin) et dans le Laboratoire de Recherche en Biologie Appliquée (LARBA) d'avril en septembre 2019. Les conditions ambiantes de la salle étaient de $26 \pm 0,5{ }^{\circ} \mathrm{C}$ et de $65,5 \pm 5 \% \mathrm{RH}$.

\subsection{Matériel animal}

\section{$>$ Elevage des larves de $P$. xylostella}

La population de $P$. xylostella a été collectée sur les sites maraîchers de Sèmé Kpodji, et de Houéyiho. Les larves et les nymphes recueillies ont été isolées dans des boîtes en plastique cylindriques $(3 \mathrm{~cm} \times 7 \mathrm{~cm})$ avec 
des couvercles percés de petits trous pour la ventilation. Les larves sont nourries avec les feuilles de la plante hôte (Brassica oleracea var. Capitata), puis suivies jusqu'à l'apparition des papillons de nuit. Les adultes émergés ont été récupérés et introduits dans une cage cubique de 500 mm de côté. Les œufs sont collectés quotidiennement sur un plant de chou qui se développe. Au quatrième stade (L4), les larves sont transférées sur des feuilles fraîches déposées au fond d'une grande boîte en plastique $(28 \mathrm{~cm} \times 27 \mathrm{~cm})$ où elles effectuent leur nymphose. Les nymphes sont collectées quotidiennement. À l'émergence, les adultes ont été placés dans la cage de nid et nourris avec de l'eau et du miel. L’élevage a été réalisé dans une pièce à conditions climatiques contrôlées: $25^{\circ} \mathrm{C} \pm, 75 \% \mathrm{HR}$. Des larves de deuxième et de troisième stades nouvellement apparues ont été utilisées pour nos différents essais du fait de leur attaque.

\subsection{Méthodes}

\subsubsection{Evaluation de l'effet du traitement sur l'appétence de $P$. xylostella}

L'appétence des larves face à différentes doses de pesticides à savoir : $100 \%, 75 \% 50 \%, 25 \%$, et $10 \%$ des extraits et le produit chimique de synthèse en première expérimentation en seconde expérimentation les doses d'application des pesticides botaniques utilisés étaient de : 50\%, 25\%,10\%, $5 \%, 3 \%, 1 \%$ et de $10 \% ; 5 \%, 3 \%, 2 \%, 1 \%$ et $0,5 \%$ pour le témoin de référencé (Lambda) sur les larves de stade 2 a été évaluée en estimant la surface foliaire consommée par les larves au bout de 24 heures. Le contrôle négatif est appliqué avec de l'eau distillée dose zéro. Chaque rondelle de feuille de chou mise en présence des larves a été retirée après 24 heures et déposée sur le papier millimétré. Les surfaces consommées ont été reproduites sur ce papier puis évaluées.

\section{4. Détermination des effets (mortalité, et répulsivités des extraits huile végétale, Topbio et Lambda ) sur les larves $P$. xylostella \\ $>$ Traitements et doses appliqués}

Pour déterminer l'effet des différents extraits sur les larves de stade 2 et 3 de $P$. xylostella, cinq pesticides botaniques et un témoin traité ( Lambda cyalothrine) ont été utilisés à différentes doses. Pour la première expérimentation, cinq doses ont été préparées comme suit: 100\%, 75\% 50\%, $25 \%$, et $10 \%$ des extraits et le produit chimique de synthèse ont été préparées avec $30 \mathrm{mg}$ de la lécithine dans un volume de $300 \mathrm{ml}$ plus le savon sans détergent dans l'eau distillée pour chaque solution mère. L'insecticide lambda a été utilisé à la même proportion que les autres extraits dans la première expérimentation. Pour la seconde expérimentation les doses d'application des pesticides botaniques utilisés étaient de : 50\%, 25\%,10\%, 5\%, 3\%, $1 \%$ et de $10 \%, 5 \%, 3 \%, 2 \%, 1 \%$ et $0,5 \%$ pour le témoin de référencé (Lambda) sur les 
larves de stade 2. Le contrôle négatif est appliqué avec de l'eau distillée. Un agent mouillant a été ajouté aux différents traitements tween 80.

\subsection{Essais biologiques de toxicité}

Le test biologique de toxicité était une méthode de trempage des feuilles similaire à celle utilisée par Tabashnik et al. (1990). Pour l'expérience de test de toxicité biologique, les larves du deuxième et troisième stade ont été traitées par application orale sur des disques de feuilles de chou. Des tissus de feuille (6 cm de diamètre) ont été coupés à partir de plants de chou non infestés produites dans la serre, Ces feuilles ont été lavées proprement à l'eau de javel $10 \%$ et rincées à l'eau de robinet. Les feuilles individuelles ont été plongées dans la solution insecticide préparée pendant 30 s et suspendues verticalement pour être sécher à la température ambiante pendant $5 \mathrm{mn}$. Dix larves de $P$. xylostella en élevage ont alors été mises sur la face supérieure des feuilles placées dans chaque boîte de Pétri $(6 \mathrm{~cm}$ x 1,5 cm), ayant une ouverture d'environ $3 \mathrm{~cm}$ sur le couvercle, recouverte de mousseline fine pour permettre l'aération, contenant un disque foliaire de $5 \mathrm{~cm}$ de diamètre découpé. Les disques foliaires ont été posés sur du coton imbibé d'eau dans des boîtes de Pétri de $8,5 \mathrm{~cm}$ de diamètre pour maintenir la fraîcheur des feuilles. Trois répétitions ont été faites pour chaque concentration d'extraits dans trois blocs aléatoires complets, soit 180 larves $\mathrm{L}_{2}$ et L3 testées par concentration. Le traitement témoin (uniquement d'eau distillée stérilisée) a été constitué de 60 larves $\mathrm{L}_{2}$ et de L3 de $P$. xylostella traitées avec une solution de tween 80 stérilisée. Les boîtes de Pétri ont été ensuite déposées dans des plateaux au laboratoire à la température variant entre $26 \pm 1^{\circ} \mathrm{C}$. La mortalité larvaire a été enregistrée toutes les 24 heures jusqu'au neuvième jour de traitement. Les larves sont considérées mortes si elles ne bougent pas lorsqu'elles sont légèrement pincées au forceps (Hill et Foster, 2000). Les disques foliaires ont été changés tous les trois jours avec des feuilles fraiches non traitées. Les taux de mortalité ont été calculés à 24 heures pour évaluer l'effet de l'aimantation sur le contrôle des larves.

\subsection{Analyse des données}

Les taux moyens de mortalité des larves, des chrysalides et d'émergence des chrysalides obtenues à la fin des observations, ont été utilisés pour tracer les courbes et faire les tableaux. Les taux de mortalité des insectes traités ont été corrigés en utilisant la formule d’Abbott (1925) :

$\mathrm{Mc} \%=\left(\mathrm{Me}-\mathrm{Mt}^{*} 100\right) /(100-\mathrm{Mt})$

$\mathbf{M c}=$ mortalité corrigée en pourcentage $\mathbf{M e}=$ mortalité de

l'échantillon testé $\mathrm{Mt}=$ mortalité dans le témoin non traité

Le taux d'émergence a été calculé sur la base du nombre de chrysalides formées dans chaque traitement. 
Après une transformation arc-sinus, une analyse de variance a été faite. Lorsque l'ANOVA a révélé des différences significatives entre traitements, le test SNK (Student-Newman Keuls), au seuil de $5 \%$, a été conduit afin de séparer les moyennes des différents traitements. Pour toutes ces analyses, le logiciel XLSTAT version 2019 a été utilisé.

Estimation de la DL50 (Dose Létale 50). L'analyse et la modélisation des données temps-dose-mortalité ont été effectuées en utilisant le modèle « Cox regression » (SPSS, 1989-2007). Les modèles de régression de Cox utilisent la fonction de risque pour estimer le risque d'échec relatif. La fonction de risque, $\mathrm{h}(\mathrm{t})$ est une évaluation de la mort potentielle d'un individu par unité de temps à un moment donné, étant donné que l'individu a survécu jusqu'à ce moment. Les modèles de Cox regression sont exprimés en termes de fonction de risque comme suit :

$h(t)=[h O(t)] e(B X)$

où $X$ représente log (dose), $B$ le coefficient de régression qui est le risque relatif (ici risque instantané de décès) associé à un traitement par rapport à un autre traitement, $e$ la base du logarithme népérien et $h 0(t)$ est la fonction de risque lorsque $X$ est égal à 0 .

La fonction cumulative de risque, $H(t)$, est liée à la fonction de survie et peut être dérivée de la fonction de survie comme suit :

$H(t)=-\ln S(t)$. (2)

La fonction de risque et la fonction de survie sont étroitement liées, et toutes les deux ont été calculées en utilisant le procédé de Cox regression (SPSS, 1989-2007).

La DL50 est définie comme la dose d'un agent (chimique ou biologique) nécessaire pour produire la mort de la moitié des organismes testés à un moment donné après application (Maddox, 1982). La DL50 peut être dérivée des équations (1) et (2) comme suit :

$X=10^{\wedge} \ln \left(\ln (0,5)-\ln \left(h^{0}(t)\right)\right) / B$.

Les intervalles de confiance pour la DL50 ont été calculés sur la base des mêmes équations, utilisant les informations suivantes : écart-type (SE) de $B$ et écart-type (SE) de $h 0(t)$.

\section{Résultats}

3.1. Effet de trois huiles végétales, du baume de cajou, du Topbio et l'insecticide chimique Lambda sur les larves de $P$. xylostella. Expérimentation I et II

Les tests ont été réalisés sur les larves de stade 2 et 3 de $P$. xylostella

Effet des traitements sur le comportement alimentaire de $P$. xylostella

Le comportement alimentaire a été exprimé chez $P$. xylostella en termes de surface foliaire moyenne consommée par les larves de 2ème et 3ème stades. Il 
a été mesuré en évaluant la surface totale de feuille consommée par chaque larve en fonction du traitement en 24 heures. Le tableau I montre que les surfaces moyennes consommées par une larve L3 de $P$. xylostella sont comprises entre 38,51 \pm 3,95, 33,87 $\pm 0,98,24,1 \pm 1,15,21,33 \pm 3,5$ 19,4 $\pm 2,12$, et $268,44 \pm 21,51 \mathrm{~mm}^{2}$ à une dose d'application de $10 \%$ respectivement pour l'huile de Thevetia, de Ricinus, de CNSL, de Tephrosia, du Topbio, et le traitement témoin $0 \%$. En ce qui concerne le tableau II, les surfaces moyennes consommées par une larve L2 de $P$. xylostella sont comprises entre $89,12 \pm 1,60$ b, 67,80 $\pm 31,22,95,88 \pm 0,88,105,84 \pm 3,11,60,01 \pm 31,88,50,33 \pm$ 27,14 et $205,44 \pm 33,54 \mathrm{~mm}^{2}$ à une dose d'application de $1 \%$ respectivement pour le CNSL, T. purpurea, $R$. communis, T. neriifolia, Topbio, Lambda cyalothrine (témoin de référence) et le témoin négatif. Les surfaces foliaires consommées par les larves de P. xylostella sont plus grandes au niveau des témoins non traités qu'au niveau des feuilles traitées. Le test de comportement alimentaire des larves a montré que la consommation des feuilles est dépendante de la dose de pesticide botanique ou chimique de synthèse utilisée. Plus la dose est élevée, moins a été la consommation observée aussi bien pour tous les extraits végétaux et l'insecticide chimique de synthèse. Les plus grandes surfaces foliaires ont été consommées chez les larves L3 au niveau des doses de $10 \%$ des biopesticides testés, alors qu'à partir de cette dose un phénomène d'inappétence a été observé chez les larves L2 avec les mêmes produits. Il existe une différence significative entre les surfaces consommées par les larves L2 de P. xylostella ( $\mathrm{F}=605,836 ; \mathrm{ddl}=41 ; \mathrm{P}<0,0001)$. Pour les doses de $100 \%, 75 \%, 50 \%$, des extraits d'huile végétale et du CNSL $25 \%$ un phénomène d'inappétence a été constaté également chez certaines larves L3 qui malgré la mise à jeun préalable au test n’ont consommé aucune partie de la feuille. Il existe également une différence significative entre les surfaces consommées par les larves L3 de P. xylostella ( $\mathrm{F}=505,836$; ddl $=41 ; \mathrm{P}<$ $0,0001)$.

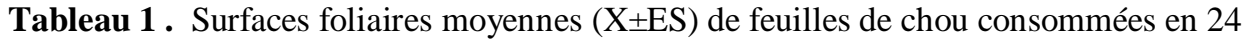
heures par les larves L3 de P. xylostella en fonction du traitement.

\section{Expérimentation I}

Stade larvaire : L3

\begin{tabular}{ccc}
\hline Traitements & Doses (\%) & Surface moyenne consommée \pm ES (mm2) \\
\hline Témoin & 0 & $268,44 \pm 21,51 \mathrm{a}$ \\
\hline CNSL à froid & 100 & $00 \pm 00 \mathrm{~d}$ \\
& 75 & $00 \pm 00 \mathrm{~d}$ \\
& 50 & $00 \pm 00 \mathrm{~d}$ \\
& 25 & $00 \pm 00 \mathrm{~d}$ \\
& 10 & $24,1 \pm 1,15 \mathrm{c}$ \\
\hline
\end{tabular}




\begin{tabular}{ccc}
\hline Huile de T. purpurea & 100 & $00 \pm 00 \mathrm{~d}$ \\
& 75 & $00 \pm 00 \mathrm{~d}$ \\
& 50 & $00 \pm 00 \mathrm{~d}$ \\
& 25 & $00 \pm 00 \mathrm{~d}$ \\
\hline Huile de $R$. Communi & 10 & $21,33 \pm 3,5 \mathrm{c}$ \\
& 100 & $00 \pm 00 \mathrm{~d}$ \\
& 75 & $00 \pm 00 \mathrm{~d}$ \\
& 50 & $00 \pm 00 \mathrm{~d}$ \\
Huile de T. neriifolia & 25 & $25 \pm 1,56 \mathrm{c}$ \\
& 10 & $33,87 \pm 0,98 \mathrm{c}$ \\
\hline Topbio & 100 & $00 \pm 00 \mathrm{~d}$ \\
& 75 & $00 \pm 00 \mathrm{~d}$ \\
& 50 & $00 \pm 00 \mathrm{~d}$ \\
& 25 & $30,17 \pm 2,14 \mathrm{c}$ \\
& 1 & $38,51 \pm 3,95 \mathrm{~b}$ \\
\hline Lambda cyalothrine & 100 & $00 \pm 00 \mathrm{~d}$ \\
& 75 & $00 \pm 00 \mathrm{~d}$ \\
& 50 & $00 \pm 00 \mathrm{~d}$ \\
& 25 & $00 \pm 00 \mathrm{~d}$ \\
& 100 & $19,4 \pm 1,15 \mathrm{c}$ \\
\hline
\end{tabular}

\section{Expérimentation II}

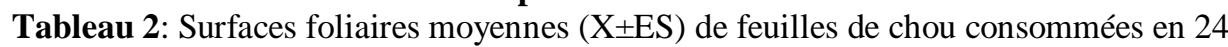
heures par les larves L2 de P. xylostella en fonction du traitement

Stade larvaire : L2

\begin{tabular}{ccc}
\hline Traitements & Doses (\%) & Surface moyenne consommée \pm ES (mm2) \\
\hline Témoin & 0 & $205,44 \pm 33,54$ \\
& & CNSL à froid \\
25 & $00 \pm 00 \mathrm{e}$ \\
10 & $00 \pm 00 \mathrm{e}$ \\
5 & $00 \pm 00 \mathrm{e}$ \\
3 & $10,14 \pm 1,11 \mathrm{~d}$ \\
1 & $21,31 \pm 18,40 \mathrm{~d}$ \\
& Huile de T. purpurea \\
50 & $00 \pm 00 \mathrm{e}$ \\
25 & $00 \pm 00 \mathrm{e}$ \\
10 & $00 \pm 00 \mathrm{e}$ \\
5 & $00 \pm 00 \mathrm{e}$ \\
3 & $17,18 \pm 77,15 \mathrm{~d}$ \\
1 & $67,80 \pm 31,22 \mathrm{c}$ \\
\hline
\end{tabular}




\begin{tabular}{ccc}
\hline Huile de $R$. Communi & 50 & $00 \pm 00 \mathrm{e}$ \\
& 25 & $00 \pm 00 \mathrm{e}$ \\
& 10 & $00 \pm 00 \mathrm{e}$ \\
& 5 & $14 \pm 1,23 \mathrm{~d}$ \\
& 3 & $25 \pm 4,14 \mathrm{~d}$ \\
& 1 & $95,88 \pm 0,88 \mathrm{~b}$ \\
\hline Huile de T. neriifolia & 50 & $00 \pm 00 \mathrm{e}$ \\
& 25 & $00 \pm 00 \mathrm{e}$ \\
& 10 & $00 \pm 00 \mathrm{e}$ \\
& 5 & $30,17 \pm 2,14 \mathrm{~d}$ \\
& 3 & $40,12 \pm 5,66 \mathrm{cb}$ \\
& 1 & $105,84 \pm 2,11 \mathrm{~b}$ \\
\hline Topbio & 50 & $00 \pm 00 \mathrm{e}$ \\
& 25 & $00 \pm 00 \mathrm{e}$ \\
& 10 & $00 \pm 00 \mathrm{e}$ \\
& 5 & $00 \pm 00 \mathrm{e}$ \\
& 3 & $19,78 \pm 3,21 \mathrm{~d}$ \\
& 1 & $60,01 \pm 1,88$ \\
\hline Lambda Cyalothrine & 10 & $00 \pm 00 \mathrm{e}$ \\
& 5 & $00 \pm 00 \mathrm{e}$ \\
& 3 & $00 \pm 00 \mathrm{e}$ \\
& 2 & $6,85 \pm 00,19 \mathrm{~d}$ \\
& 1 & $40,31 \pm 74,94 \mathrm{cb}$ \\
& 0,5 & $50,33 \pm 27,14 \mathrm{c}$ \\
\hline
\end{tabular}

$\mathrm{ES}=$ Erreur Standard

Les moyennes suivies des mêmes lettres ne sont pas significativement différentes au seuil de 5\% (ANOVA suivie du test de Student-Newman-Keuls).

\section{3 .2. Effet direct de la consommation de feuilles traitées sur la mortalité larvaire des larves de stade L2 et L3 de P. xylostella. Expérimentation I et II}

L'exposition des larves de $P$. xylostella aux feuilles de chou traitées avec différentes doses des biopesticides et du pesticide de synthèse pendant 24 heures a entraîné des taux moyens de mortalité présentés sur les figures (1 à 12). Le taux de mortalité a été croissant pour toutes les doses du 1er au neuvième jour. Le produit chimique de synthèse Lambda a éliminé 6 jours après traitement, $100 \%$ de larves L3 de $P$. xylostella aux doses respectives de $100 \%, 75 \%, 50 \%, 25 \%$, et $10 \%$ pendant la première expérimentation. Alors qu'en deuxième expérimentation 100\% de mortalité ont été enregistrées à une dose d'application de 5\%. Ces taux de mortalité étaient similaires aux celles des doses de $50 \%$, et $25 \%$ en première expérimentation et $10 \%$ en deuxième expérimentation respectivement pour les huiles végétales, CNSL et Topbio. Pour la dose d'application de $10 \%$ d'huile de $T$. purpurea, le CNSL, l'huile de $R$. communis, et de $T$. neriifolia ; les mortalités cumulées étaient de 74,11\%, 64,33\%, 62,60\%, 50,45\% de larves L3 de P. xylostella. Avec les larves de stade 2 de $P$. xylostella, les mortalités cumulées étaient de 78,15\%, 72,68\%, 
55,21\%, 51,77\%, 100\% respectivement pour T. purpurea, CNSL, $R$. communis, T. neriifolia, et Topbio à une dose d'application de $5 \%$. A cette dose le taux de mortalité était similaire aux doses de 10\% des mêmes produits sur les larves de stade3. Comparé au témoin non traité, toutes les doses utilisées ont entrainé une réduction hautement significative de la population de P. xylostella après 9 jours $(\mathrm{F}=509,78 ; \mathrm{ddl}=41 ; \mathrm{P}<.0001)$. L'analyse de variance suivie du test de SNK a montré que les mortalités cumulées des larves, pour les doses 100\%, 75\% 50\% et 25\% de, CNSL à froid et les extraits d'huile végétale, se sont significativement distinguées de celles de $10 \%$ et de $5 \%$ respectivement pour le stade 3 et 2 . Par rapport au témoin de référence Lambda cyalothrine, les mortalités cumulées des doses de $25 \%$ et $10 \%$ se sont également distinguées significativement des doses de $25 \%$ et $10 \%$ des autres pesticides botaniques utilisés. Tout comme pour le témoin de référence et les autres pesticides botaniques les taux de mortalités étaient élevés pour le $2^{\text {eme }}$ stade comparativement à ceux obtenus avec le $3^{\text {eme }}$ stade exposé aux mêmes produits. Il y a une différence significative entre les produits utilisés $\mathrm{P}<0001$.

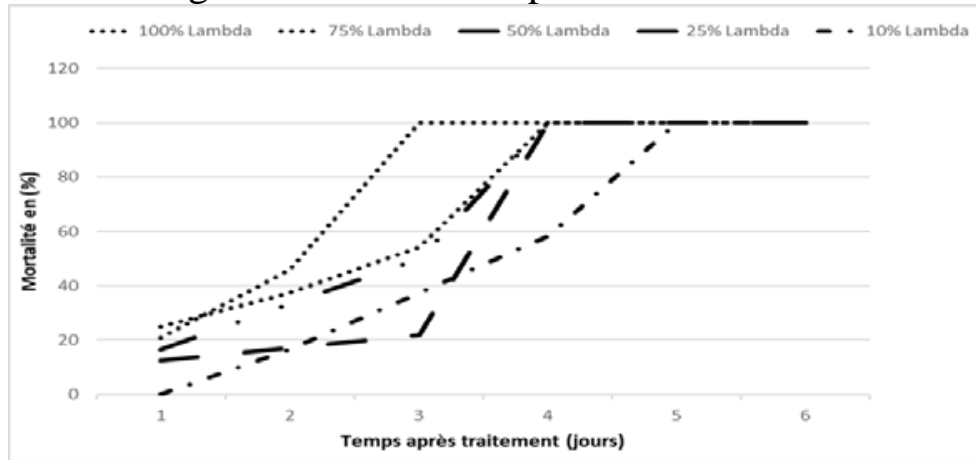

Figure 1 : Taux de mortalité des larves L3 de P. xylostella après application de différentes doses de Lambda cyalothrine.

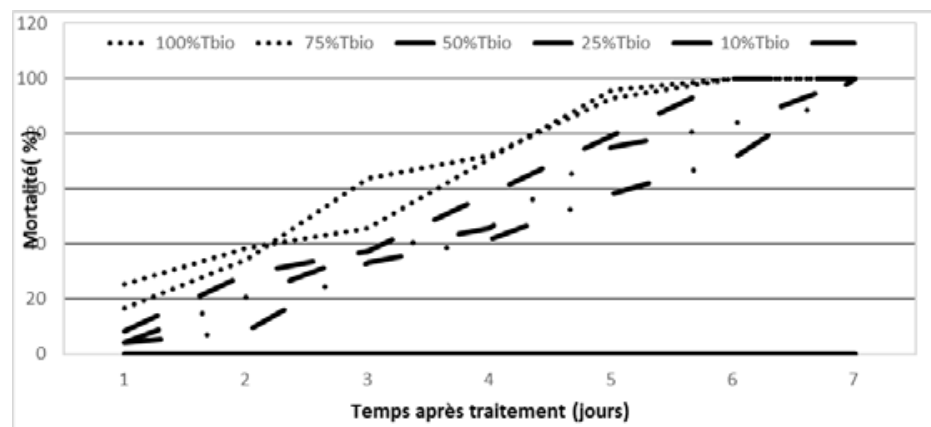

Figure 2 : Taux de mortalité des larves L3 de P. xylostella après application de différentes doses de Topbio 


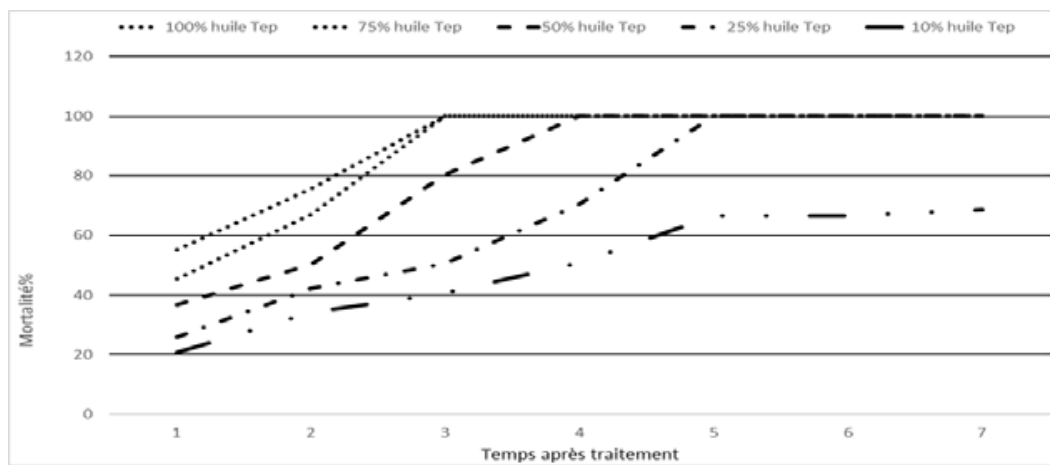

Figure 3 : Taux de mortalité des larves L3 de P. xylostella après application de différentes doses d'huile de Tephrosia purpurea

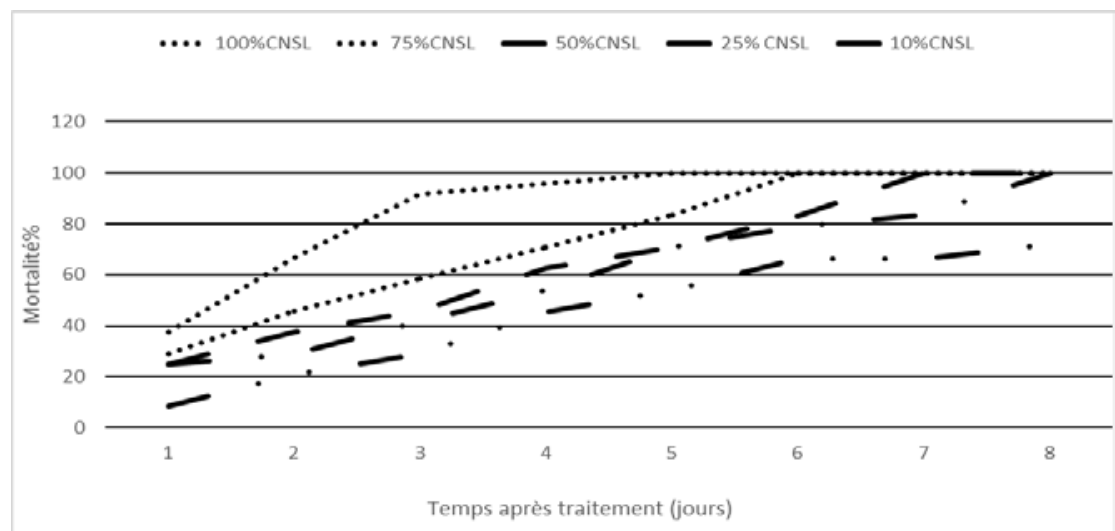

Figure 4 : Taux de mortalité des larves L3 de P. xylostella après application de différentes doses d' huile de CNSL à froid .

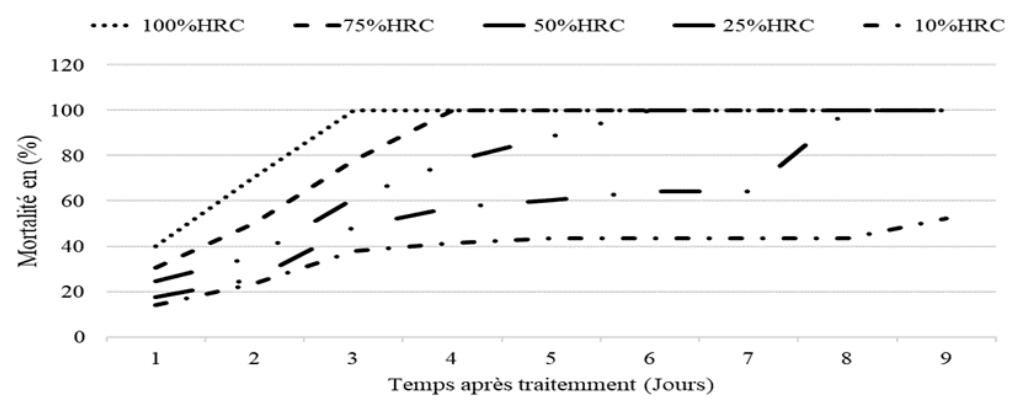

Figure 5 : Taux de mortalité des larves L3 de P. xylostella après application de différentes doses d'huile de $R$. Communis 


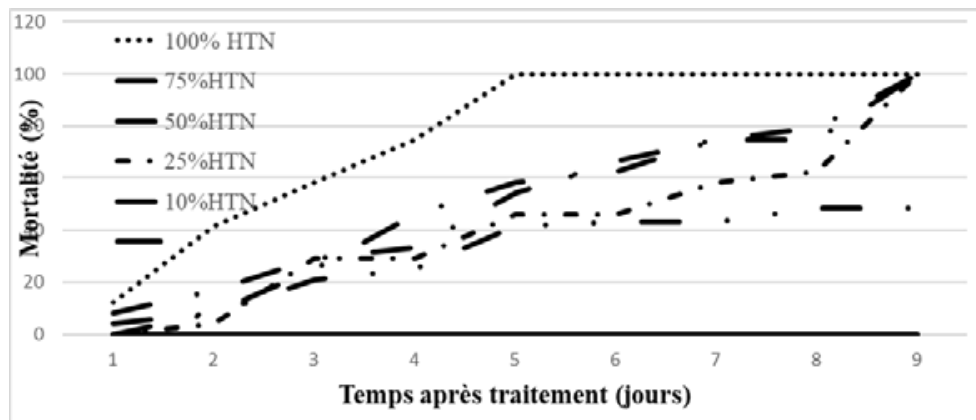

Figure 6 : Taux de mortalité des larves L3 de P. xylostella après application de différentes doses d' huile de T. Neriifolia

\section{Expérimentation II}

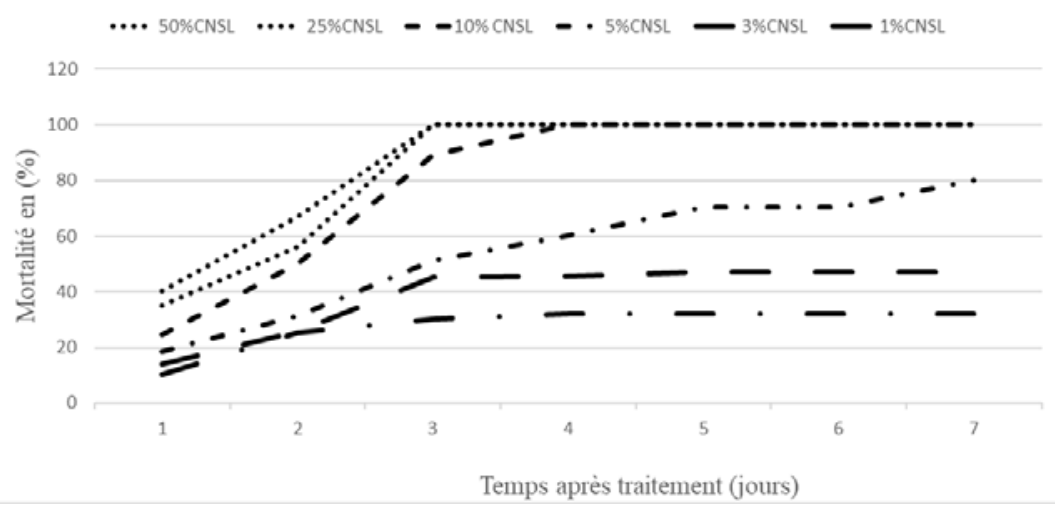

Figure 7 : Taux de mortalité des larves L2 de P. xylostella après application de différentes doses d' huile de CNSL à froid

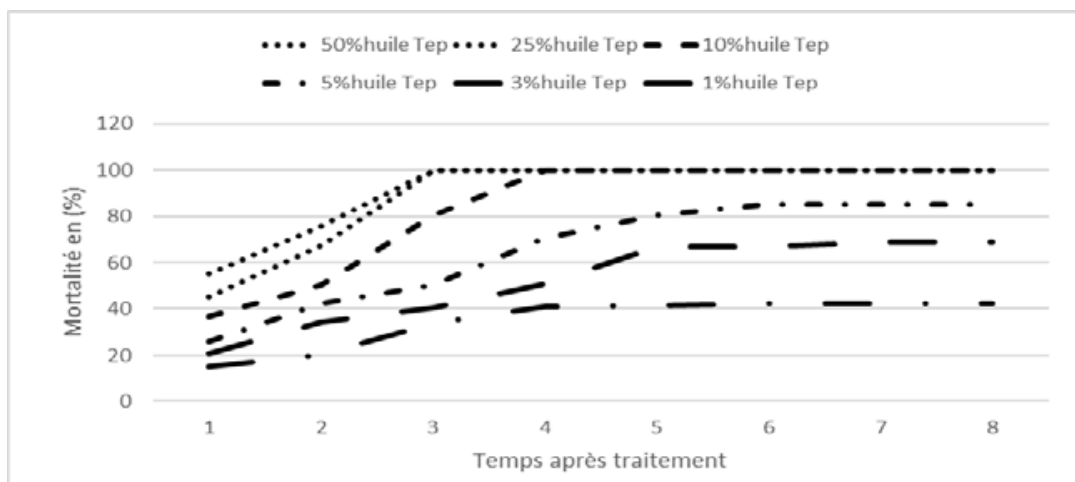

Figure 8 : Taux de mortalité des larves L2 de $P$. xylostella après application de différentes doses d’huile de Tephrosia purpurea 


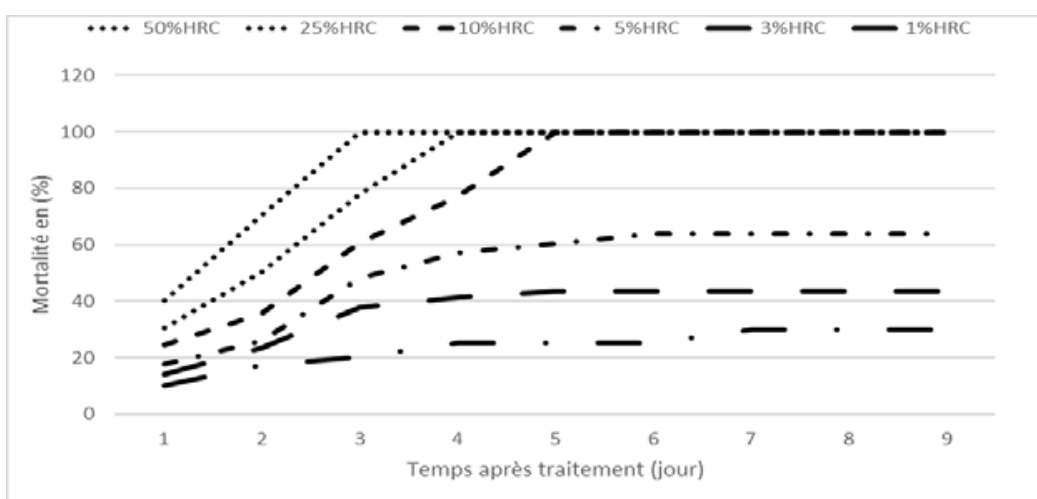

Figure 9 : Taux de mortalité des larves L2 de P. xylostella après application de différentes doses d'huile de Ricinus communis

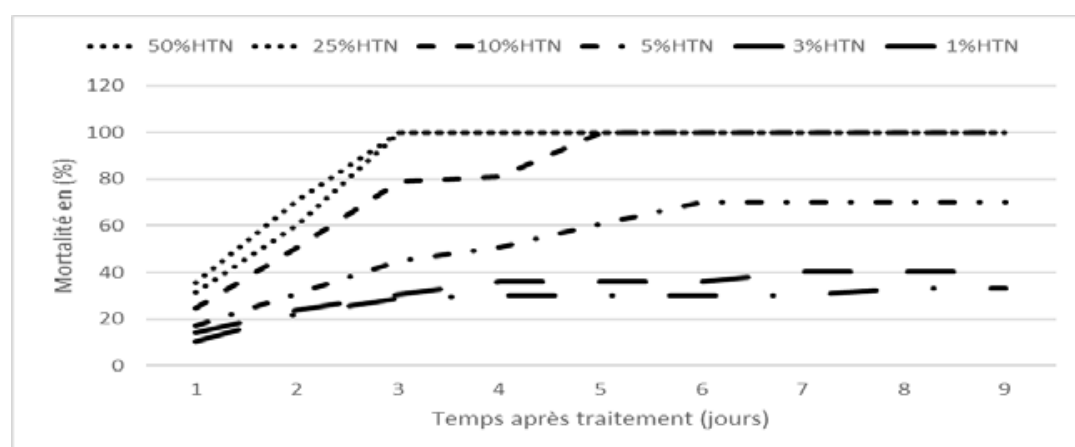

Figure 10 : Taux de mortalité des larves L2 de P. xylostella après application de différentes doses d'huile de T. Neriifolia

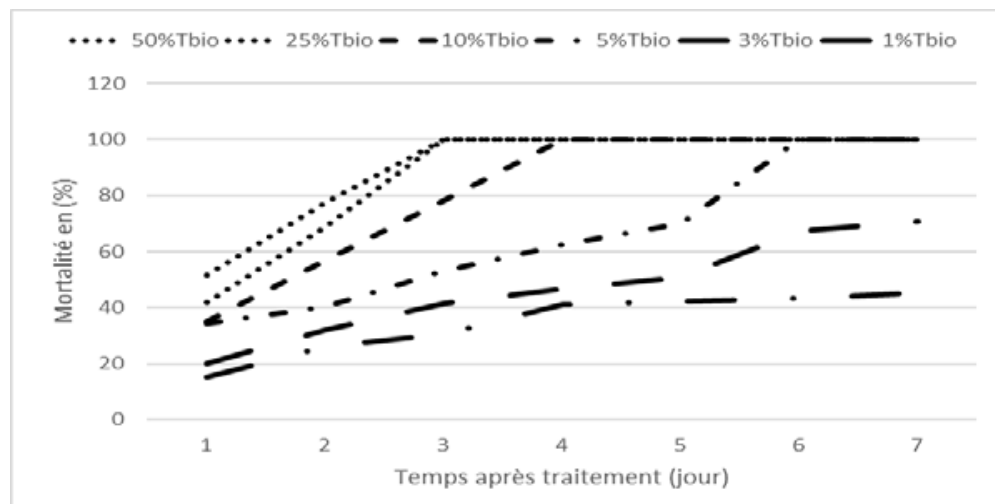

Figure 11 : Taux de mortalité des larves L2 de P. xylostella après application de différentes doses de Topbio. 


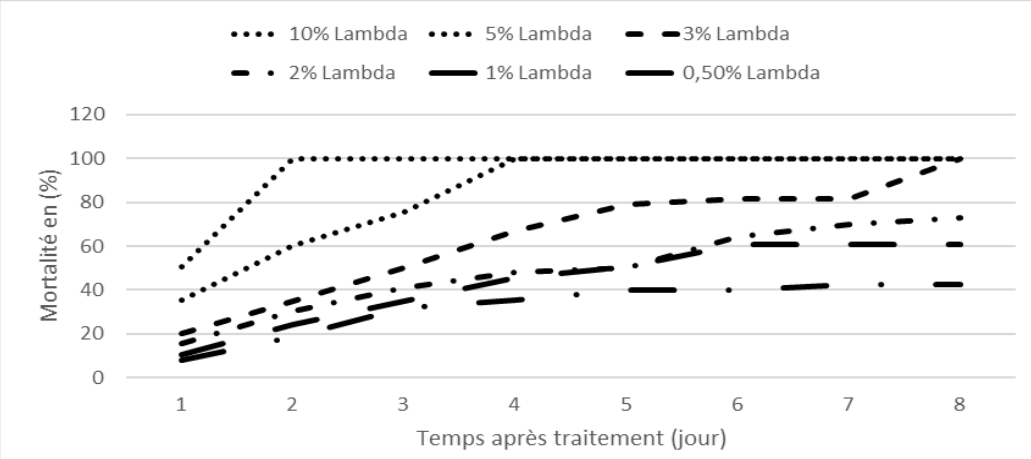

Figure 12 : Taux de mortalité des larves L2 de P. xylostella après application de différentes doses de Lambda cyalothrine

\subsection{Variation du taux de mortalité et d'émergence des larves L2 et L3 de P. xylostella \\ Expérimentation I et II}

Après avoir exposé les larves du stade 3 de l'espèce $P$. xylostella aux différentes concentrations des trois huiles végétales, du baume de cajou, du Topbio et de l'insecticide de synthèse Lambda pendant $24 \mathrm{~h}$, le taux de mortalité et d'émergence varient selon les concentrations (Tableau 3). Pour tous les extraits, la mortalité des larves a atteint un taux de $100 \%$ à partir d'une concentration de $25 \%$. En ce qui concerne, les deux témoins de référence Lambda et Topbio la mortalité est plafonnée à $100 \%$ dès la concentration de $10 \%$. Il existe une différence significative entre les larves traitées à une dose d'application de $1 \%$ et le témoin non traité. Il existe également une différence significative entre les plus fortes doses (100\%, $75 \%, 50 \%$ et $25 \%)$ et les doses les plus faibles $(10 \%, 5 \%, 3 \%$ et $1 \%)$. De l'ensemble de ces résultats un premier classement de l'efficacité toxique des extraits testés est mis en évidence, ainsi les extraits les plus toxiques sont ceux des T. purpurea, CNSL, et $R$. communis, et le moins toxique celui de $T$. neriifolia. Une analyse comparative de la mortalité larvaire induite par les différents extraits des pesticides botaniques montre une plus grande efficacité des huiles de Tephrosia et du CNSL ( $\mathrm{p}<0.05)$, même à une dose de $5 \%$ de la solution usitée, le taux de mortalité va au-delà de $60 \%$ après application sur les larves L2 ( tableau 3). Des mortalités intermédiaires sont induites par les huiles végétales de $R$. communis et de T. neriifolia. Cependant, elle provoque la mortalité larvaire la plus faible. Le temps d'exposition varie aussi en fonction du pesticide et de la dose utilisé pour les différents traitements. Les résultats d'analyse de la variance relatifs à l'effet insecticide des huiles de plantes sur $P$. xylostella révèlent que le taux de mortalité a une variation très hautement significative suivant la dose, le stade larvaire et le temps d'exposition considéré d'une part, selon la plante, le stade larvaire et le temps d'exposition 
d'autre part ( $\mathrm{P}<0,001)$. Cela implique que l'effet insecticide observé dépend de la nature de la plante, de la dose et du stade larvaire du stade (Tableau 3 et 4).

Tableau 3 : Mortalité (\%) des larves L3 de P. xylostella en fonction de la dose des extraits d'huile (\%) de trois espèces végétales, du CNSL à froid, Topbio et le produit chimique de synthèse lambda après 24 heures d'exposition aux feuilles de chou traitées.

\begin{tabular}{|l|l|l|l|l|l|l|}
\hline \multicolumn{7}{|l|}{ Pourcentage moyen de mortalités des larves L3 de P. xylostella } \\
\hline $\begin{array}{l}\text { Doses } \\
\text { Pesticides }\end{array}$ & $100 \%$ & $75 \%$ & $50 \%$ & $25 \%$ & $10 \%$ & Témoin \\
\hline $\begin{array}{l}\text { Tephrosia } \\
\text { purpurea }\end{array}$ & $100 \pm 0 \mathrm{a}$ & $100 \pm 0 \mathrm{a}$ & $100 \pm 0 \mathrm{a}$ & $100 \pm 0 \mathrm{a}$ & $78,10 \pm 4,3 \mathrm{~b}$ & $6,51 \pm 1,28 \mathrm{~d}$ \\
\hline $\begin{array}{l}\text { CNSL à } \\
\text { froid }\end{array}$ & $100 \pm 0 \mathrm{a}$ & $100 \pm 0 \mathrm{a}$ & $100 \pm 0 \mathrm{a}$ & $100 \pm 0 \mathrm{a}$ & $74,19 \pm 3,7 \mathrm{~b}$ & $5,61 \pm 1,01 \mathrm{~d}$ \\
\hline $\begin{array}{l}\text { Ricinus } \\
\text { communis }\end{array}$ & $100 \pm 0 \mathrm{a}$ & $100 \pm 0 \mathrm{a}$ & $100 \pm 0 \mathrm{a}$ & $100 \pm 0 \mathrm{a}$ & $69,09,45 \pm 5,61 \mathrm{bc}$ & $5,56 \pm 1,03 \mathrm{~d}$ \\
\hline $\begin{array}{l}\text { Thvethia } \\
\text { neriifolia }\end{array}$ & $\begin{array}{c}100 \pm 0 \\
\mathrm{a}\end{array}$ & $100 \pm 0 \mathrm{a}$ & $100 \pm 0 \mathrm{a}$ & $100 \pm 0 \mathrm{a}$ & $62,77 \pm 3,59 \mathrm{bc}$ & $4,45 \pm 1,13 \mathrm{~d}$ \\
\hline $\begin{array}{l}\text { Topbio } \\
100 \pm 0 \\
\mathrm{a}\end{array}$ & $100 \pm 0 \mathrm{a}$ & $100 \pm 0 \mathrm{a}$ & $100 \pm 0 \mathrm{a}$ & $100 \pm 0 \mathrm{a}$ & $6,07 \pm 1,35 \mathrm{~d}$ \\
\hline $\begin{array}{l}\text { Lambda } \\
\text { Cyalothrine }\end{array}$ & $100 \pm 0 \mathrm{a}$ & $100 \pm 0 \mathrm{a}$ & $100 \pm 0 \mathrm{a}$ & $100 \pm 0 \mathrm{a}$ & $100 \pm 0 \mathrm{a}$ & $3,97 \pm 8,16 \mathrm{~d}$ \\
\hline
\end{tabular}

\section{Expérimentation II}

Tableau 4 : Mortalité (\%) des larves L2 de P. xylostella en fonction de la dose des extraits d'huile (\%) de trois espèces végétales, du CNSL à froid, Topbio et le produit chimique de synthèse lambda après 24 heures d'exposition aux feuilles de chou traitées

\begin{tabular}{|l|l|l|l|l|l|l|}
\hline \multicolumn{6}{|l|}{ Pourcentage moyen de mortalités des larves L2 de P. xylostella } \\
\hline $\begin{array}{l}\text { Doses } \\
\text { Pesticides }\end{array}$ & $25 \%$ & $10 \%$ & $5 \%$ & $3 \%$ & $1 \%$ & Témoin \\
\hline $\begin{array}{l}\text { Tephrosia } \\
\text { purpurea }\end{array}$ & $100 \pm 0$ a & $100 \pm 0 \mathrm{a}$ & $78,15 \pm 3,1 \mathrm{~b}$ & $51,12 \pm 3,10 \mathrm{bc}$ & $30,10 \pm 6,3 \mathrm{~cd}$ & $5,91 \pm 7,2 \mathrm{e}$ \\
\hline $\begin{array}{l}\text { CNSL à } \\
\text { froid }\end{array}$ & $100 \pm 0$ a & $100 \pm 0 \mathrm{a}$ & $72,68 \pm 4,6 \mathrm{~b}$ & $49,22 \pm 7,7$ bc & $25,76, \pm 2,1 \mathrm{~cd}$ & $4,30 \pm 3,03 \mathrm{e}$ \\
\hline $\begin{array}{l}\text { Ricinus } \\
\text { communis }\end{array}$ & $100 \pm 0 \mathrm{a}$ & $100 \pm 0 \mathrm{a}$ & $\begin{array}{l}55,21 \pm 6,31 \mathrm{~b} \\
\mathrm{c}\end{array}$ & $46,85 \pm 5,6 \mathrm{bc}$ & $18,03 \pm 4,3 \mathrm{~cd}$ & $4,44 \pm 1,02 \mathrm{e}$ \\
\hline $\begin{array}{l}\text { Thvethia } \\
\text { neriifolia }\end{array}$ & $\begin{array}{l}100 \pm 0 \\
\mathrm{a}\end{array}$ & $100 \pm 0 \mathrm{a}$ & $\begin{array}{l}51,77 \pm 2,5 \mathrm{ab} \\
\mathrm{c}\end{array}$ & $27,61 \pm 4,3 \mathrm{~cd}$ & $15,11 \pm 3,4 \mathrm{~cd}$ & $3,67 \pm 2,3 \mathrm{e}$ \\
\hline $\begin{array}{l}\text { Topbio } \\
100 \pm 0\end{array}$ & $100 \pm 0 \mathrm{a}$ & $100 \pm 0 \mathrm{a}$ & $56,84 \pm 2,7$ abc & $33,16 \pm 6,33 \mathrm{bc}$ & $5,74 \pm 1,45 \mathrm{e}$ \\
\hline $\begin{array}{l}\text { Lambda } \\
\text { Cyalothrine }\end{array}$ & $100 \pm 0 \mathrm{a}$ & $100 \pm 0 \mathrm{a}$ & $100 \pm 0 \mathrm{a}$ & $81,67 \pm 3,1 \mathrm{~b}$ & $46,39 \pm 5,11 \mathrm{abc}$ & $4,41 \pm 9,13 \mathrm{e}$ \\
\hline
\end{tabular}


Les moyennes suivies de lettres différentes sont significativement différentes au seuil de 5\% (ANOVA suivie du test de Student-Newman-Keuls)

\subsection{Effet des traitements sur la formation des chrysalides et le taux d'émergence des adultes de $P$. xylostella issus des larves $\mathrm{L} 2$ et $\mathrm{L} 3$ traitées}

Le tableau 5, montre que les taux de chrysalidation de $P$. xylostella par rapport aux larves survivantes après $24 \mathrm{~h}$ d'exposition aux feuilles de chou traitées étaient plus faibles pour les doses d'application de $10 \%$ de CNSL, de $T$. purpurea, $R$. communis de T. neriifolia, et du Topbio (18,7 \%, 17,77 \%, 28,4 $\%$, 30\%, et $14,15 \%$ de chrysalides respectivement) sur les larves L3 comparé aux taux de chrysalidation obtenus des doses de $1 \%$ des mêmes produits sur les larves L2 ( 44,12 ,33,66\%, 52,45\%, 62,14\% et 42,77\% respectivement). Les taux d'émergence des individus ayant survécu aux différents traitements ont varié de $50,2 \%$ à $77,8 \%$ à une dose d'application de $10 \%$ sur les larves L3 et de $75,42 \%$ à $94,10 \%$ à une dose d'application de $1 \%$ sur les larves L2. L'émergence des femelles était supérieure à celle des mâles

Tableau 5: Taux moyens de chrysalidation, d'adultes de $P$. xylostella émergés après application de différentes doses de CNSL, Tephrosia purpurea, Ricinus communis, thevetia neriifolia, Topbio et Lambda

\begin{tabular}{|c|c|c|c|c|c|c|c|c|}
\hline Traitement & $\begin{array}{c}\text { Stades } \\
\text { Lavaires( } \\
\text { L2 L3) }\end{array}$ & $\begin{array}{c}\text { Dose } \\
\%\end{array}$ & $\begin{array}{c}\% \text { de } \\
\text { chrysalidation }\end{array}$ & $\begin{array}{c}\text { Taux } \\
\text { d'emergence } \\
\text { des adultes } \\
\%\end{array}$ & $\begin{array}{c}\text { Nombre } \\
\text { de } \\
\delta\end{array}$ & $\begin{array}{c}\text { Nombre } \\
\text { de } \\
q \\
q\end{array}$ & $\begin{array}{c}q \\
\text { Malformées }\end{array}$ & $\begin{array}{c}c \hat{\jmath} \\
\text { Malformés }\end{array}$ \\
\hline Témoin & & 0 & 96,4 & 95,12 & 31 & 19 & 0 & 0 \\
\hline \multirow[t]{4}{*}{ CNSL } & L3 & 10 & 18,7 & 61,4 & 1 & 3 & 1 & 0 \\
\hline & \multirow{3}{*}{ L2 } & 5 & 12,16 & 72,32 & 6 & 2 & 1 & 0 \\
\hline & & 3 & 18,44 & 78,39 & 7 & 4 & 2 & 1 \\
\hline & & 1 & 44,12 & 89,14 & 10 & 6 & 0 & 0 \\
\hline \multirow{3}{*}{$\begin{array}{l}\text { Tephrosia } \\
\text { Purpurea }\end{array}$} & L3 & 10 & 17,77 & 50,2 & 2 & 2 & 0 & 1 \\
\hline & \multirow[t]{2}{*}{ L2 } & 3 & 15,78 & 78,14 & 7 & 4 & 5 & 3 \\
\hline & & 1 & 33,66 & 84,04 & 9 & 5 & 0 & 1 \\
\hline \multirow{4}{*}{$\begin{array}{c}\text { Ricinus } \\
\text { communis }\end{array}$} & L3 & 10 & 28,4 & 67,78 & 3 & 4 & 1 & 0 \\
\hline & \multirow{3}{*}{ L2 } & 5 & 17,60 & 64,33 & 7 & 3 & 3 & 2 \\
\hline & & 3 & 40,05 & 78,84 & 9 & 4 & 3 & 0 \\
\hline & & 1 & 52,45 & 91,33 & 11 & 6 & 8 & 5 \\
\hline \multirow{4}{*}{$\begin{array}{l}\text { Thevetia } \\
\text { neriifolia }\end{array}$} & L3 & 10 & 30 & 77,8 & 7 & 5 & 2 & 1 \\
\hline & \multirow{3}{*}{ L2 } & 5 & 22,78 & 70,45 & 8 & 4 & 4 & 2 \\
\hline & & 3 & 50,55 & 77,14 & 10 & 6 & 3 & 1 \\
\hline & & 1 & 62,14 & 94,10 & 12 & 7 & 2 & 3 \\
\hline \multirow[t]{2}{*}{ Topbio } & \multirow[t]{2}{*}{$\mathrm{L} 2$} & 3 & 25,9 & 70,18 & 8 & 5 & 3 & 1 \\
\hline & & 1 & 42,77 & 80,17 & 10 & 3 & 0 & 0 \\
\hline Lambda & & 3 & 8,16 & 51,02 & 4 & 0 & 1 & 0 \\
\hline
\end{tabular}




\begin{tabular}{|l|c|c|c|c|c|c|c|c|}
\hline Cyalothrine & \multirow{2}{*}{ L2 } & 2 & 21,36 & 64,35 & 4 & 2 & 2 & 1 \\
\cline { 3 - 9 } & & 1 & 36,31 & 75,42 & 5 & 4 & 0 & 3 \\
\cline { 3 - 9 } & & 0,5 & 65,96 & 91,23 & 11 & 5 & 0 & 0 \\
\hline
\end{tabular}

+ : femelle ; ô : mâle.

3.5. Activité larvicide des extraits botaniques (Topbio, l'huile de Téphrosia, l'huile de Ricinus, l'huile de thevetia, le CNSL à froid) et l'insecticide chimique lambda sur $\boldsymbol{P}$. xylostella.

Les valeurs de DL50 et les intervalles de confiances pour chaque pesticide botanique sont présentés dans le tableau 6 . De ces résultats, il ressort que la mortalité était dépendante de la dose et du temps après exposition des larves L2 et L3 aux différents pesticides. Pour une durée d'observation de 120 heures (5 jours), la DL50 du Topbio était de $3 \%$ contre $12,5 \%$ pour 48 heures (2 jours) et 5,1\% pour 96 heures (4 jours) alors que les DL50 du témoin de référence Lambda cyalothrine étaient de $3,1 \%, 1,2 \%$ et de $5,6 \%$ et 1,9\% respectivement sur les larves L2 et L3 pour 48 heures (2 jours ) et 120 heures (5 jours). Les DL50 obtenues avec l'huile de T. purpurea, le CNSL, l'huile de ricinus et de Thevetia étaient également dépendantes de la dose et du temps. De, 13,4\%, 13,8 \%, 15,6\%, 16,9\% respectivement pour l'huile de $T$. purpurea, CNSL, Ricinus et $T$. neriifolia à 48 heures (2 jours) sur les larves L3. La DL50 pour ces mêmes pesticides botaniques pour le contrôle des larves L2 de $P$. xylostella est passé à 8,6\%, 8,1\%, 9,3\%, $14,7 \%$ et de $15,5 \%$ au bout de 48 heures ( 2 jours) alors qu'il fallait 1,4\%, 2\%, 2,4\%, 2,8\% et 3,1\% pour contrôler 50\% de ces larves en 120 heures (5 jours) respectivement pour Topbio, Tephrosia, CNSL, Ricinus et Thevetia. Considérant les DL50 et les intervalles de confiances aux différents temps après traitement, pour un même temps, la DL50 de Thevetia a été plus élevée que celles des autres . Topbio a été plus efficace que les autres extraits végétaux. Ceci se remarque également par la diminution de la largeur des intervalles de confiance, comparativement aux celles des intervalles de confiance de T. purpurea, CNSL, $R$. communis et T. neriifolia. Ces valeurs montrent l'existence d'une relation dose-réponse dans le temps pour les cinq pesticides botaniques. Le témoin de référence Lambda cyalothrine $a$ donné la valeur $B(68,287)$ la plus élevée (Tableau 10) et par conséquent, les valeurs de DL50 les moins élevées (Tableau 8). La relation dose-réponse a été très bonne pour Lambda cyalothrine (Figure 2 ). Parmi tous les pesticides botaniques le Topbio a donné la valeur de B $(14,254)$ la plus élevée (Tableau 9) et les valeurs de DL50 les plus faibles (Tableau 9 ). Les valeurs de B pour tous les pesticides sont positives cela explique que la relation dose réponse a été très bonne pour tous les pesticides utilisés (Tableau 9et 10). 
Tableau 7: Toxicité des pesticides botaniques sur les larves L3 de P. Xylostella

\begin{tabular}{|c|c|c|c|c|}
\hline Produits & $\begin{array}{l}\text { Temps après } \\
\text { traitement } \\
\text { (heures) }\end{array}$ & $\begin{array}{l}\text { DL50 (\% de } \\
\text { dilution) }\end{array}$ & $\begin{array}{l}\text { IC (Intervalles de } \\
\text { confiances) } \%\end{array}$ & P ( Probabilité) \\
\hline \multirow{5}{*}{ Lambda } & 24 & 7,4 & {$[8,1 \quad 7,3]$} & \multirow{5}{*}{$<0,001$} \\
\hline & 48 & 5,6 & {$[7,6$ 5,4 $]$} & \\
\hline & 72 & 3,7 & {$\left[\begin{array}{ll}4,2 & 3,5\end{array}\right]$} & \\
\hline & 96 & 3,1 & {$\left[\begin{array}{ll}3,5 & 2,7\end{array}\right]$} & \\
\hline & 120 & 1,9 & {$[2,6$} & \\
\hline \multirow[t]{5}{*}{ Topbio } & 24 & 20,2 & {$[24,3 \quad 18,1]$} & \multirow{5}{*}{$<0,001$} \\
\hline & 48 & 12,5 & {$\left[\begin{array}{ll}17,8 & 9,9]\end{array}\right.$} & \\
\hline & 72 & 7,3 & {$\left[\begin{array}{ll}9,9 & 7,5\end{array}\right]$} & \\
\hline & 96 & 5,1 & {$[6,8$} & \\
\hline & 120 & 3,0 & {$\left[\begin{array}{ll}3,2 & 2,4\end{array}\right]$} & \\
\hline \multirow{5}{*}{ Tephrosia } & 24 & 24,1 & {$\left[\begin{array}{ll}28,5 & 27,0\end{array}\right]$} & \multirow{5}{*}{$<0,001$} \\
\hline & 48 & 13,4 & {$\left[\begin{array}{ll}16,6 & 10,1]\end{array}\right.$} & \\
\hline & 72 & 7,8 & {$\left[\begin{array}{ll}9,8 & 7,2\end{array}\right]$} & \\
\hline & 96 & 6,0 & {$\left[\begin{array}{lll}7,2 & 5,7\end{array}\right]$} & \\
\hline & 120 & 4,3 & {$\left[\begin{array}{ll}5,3 & 4,1\end{array}\right]$} & \\
\hline \multirow[t]{5}{*}{ CNSL à froid } & 24 & 25,4 & {$[29,4 \quad 26,2]$} & \multirow{5}{*}{$<0,001$} \\
\hline & 48 & 13,8 & {$\left[\begin{array}{ll}16,8 & 10,7] \\
\end{array}\right.$} & \\
\hline & 72 & 8,1 & {$\left[\begin{array}{ll}9,9 & 7,6\end{array}\right]$} & \\
\hline & 96 & 5,8 & {$[6,6$} & \\
\hline & 120 & 4,7 & {$\left[\begin{array}{ll}5,5 & 4,5\end{array}\right]$} & \\
\hline \multirow{5}{*}{$\begin{array}{l}\text { Ricinus } \\
\text { communis }\end{array}$} & 24 & 27,2 & {$\left[\begin{array}{ll}33,1 & 32,4]\end{array}\right.$} & \multirow{5}{*}{$<0,001$} \\
\hline & 48 & 15,6 & {$\left[\begin{array}{ll}18,5 & 153\end{array}\right]$} & \\
\hline & 72 & 8,9 & {$\left[\begin{array}{ll}11,4 & 9,3\end{array}\right]$} & \\
\hline & 96 & 6,2 & $7,0]$ & \\
\hline & 120 & 5,4 & $5,7]$ & \\
\hline \multirow{5}{*}{$\begin{array}{l}\text { Thevetia } \\
\text { neriifolia }\end{array}$} & 24 & 29,4 & {$[36,4$} & \multirow{5}{*}{$<0,001$} \\
\hline & 48 & 16,9 & $13,1]$ & \\
\hline & 72 & 10,1 & {$\left[\begin{array}{ll}11,4 & 9,0\end{array}\right]$} & \\
\hline & 96 & 7,7 & {$\left[\begin{array}{lll}8,1 & 7,7 & ]\end{array}\right.$} & \\
\hline & 120 & 6,0 & {$\left[\begin{array}{ll}7,0 & 6,2\end{array}\right]$} & \\
\hline
\end{tabular}

Tableau 8: Toxicité des pesticides botaniques sur les larves L2 de P. Xylostella

\begin{tabular}{|c|c|c|c|c|}
\hline Produits & $\begin{array}{c}\text { Temps après } \\
\text { traitement } \\
\text { (heures) }\end{array}$ & $\begin{array}{c}\text { (DL50 \% de } \\
\text { dilution) }\end{array}$ & IC (Intervalles de confiances) \% & $\begin{array}{c}\mathrm{P(}( \\
\text { Probabili } \\
\text { té) }\end{array}$ \\
\hline \multirow{5}{*}{ Lambda } & 24 & 4,3 & {$\left[\begin{array}{ll}5,0 & 3,8\end{array}\right]$} & \multirow{5}{*}{$<0,001$} \\
\hline & 48 & 3,1 & {$\left[\begin{array}{ll}3,6 & 2,8\end{array}\right]$} & \\
\hline & 72 & 2,1 & {$\left[\begin{array}{ll}2,4 & 1,8\end{array}\right]$} & \\
\hline & 96 & 1,7 & {$\left[\begin{array}{ll}1,9 & 1,5\end{array}\right]$} & \\
\hline & 120 & 1,2 & {$\left[\begin{array}{ll}1,4 & 1,1\end{array}\right]$} & \\
\hline \multirow[t]{3}{*}{ Topbio } & 24 & 15,9 & {$[18,8$} & \\
\hline & 48 & 8,6 & {$[10,2$} & \\
\hline & 72 & 4,2 & {$\left[\begin{array}{ll}5,0 & 3,7\end{array}\right]$} & \\
\hline
\end{tabular}




\begin{tabular}{|c|c|c|c|c|}
\hline & 96 & 2,1 & {$[2,5 \quad 1,8]$} & \multirow[t]{2}{*}{$<0,001$} \\
\hline & 120 & 1,4 & {$[1,6$} & \\
\hline \multirow{5}{*}{ Tephrosia } & 24 & 16,2 & {$[19,9 \quad 14,1]$} & \multirow{5}{*}{$<0,001$} \\
\hline & 48 & 8,1 & {$[9,46 \quad 7,6]$} & \\
\hline & 72 & 3,7 & {$\left[\begin{array}{ll}4,31 & 3,2\end{array}\right]$} & \\
\hline & 96 & 2,7 & {$\left[\begin{array}{ll}2,9 & 2,1]\end{array}\right.$} & \\
\hline & 120 & 2,0 & {$\left[\begin{array}{ll}1,8 & 1,3\end{array}\right]$} & \\
\hline \multirow[t]{5}{*}{ CNSL à froid } & 24 & 17,2 & {$[20,414,9]$} & \multirow{5}{*}{$<0,001$} \\
\hline & 48 & 9,3 & {$\left[\begin{array}{ll}11,0 & 8,0]\end{array}\right.$} & \\
\hline & 72 & 4,2 & {$[5,0$} & \\
\hline & 96 & 3,3 & {$[2,8$} & \\
\hline & 120 & 2,4 & {$\left[\begin{array}{ll}2,0 & 1,7\end{array}\right]$} & \\
\hline \multirow{5}{*}{$\begin{array}{l}\text { Ricinus } \\
\text { communis }\end{array}$} & 24 & 20,5 & {$[24,117,18]$} & \multirow{5}{*}{$<0,001$} \\
\hline & 48 & 14,7 & {$\left[\begin{array}{ll}17,3 & 12,8\end{array}\right.$} & \\
\hline & 72 & 8,1 & {$\left[\begin{array}{lll}9,5 & 7,0\end{array}\right]$} & \\
\hline & 96 & 3,8 & {$\left[\begin{array}{ll}4,5 & 3,3\end{array}\right]$} & \\
\hline & 120 & 2,8 & {$\left[\begin{array}{lll}3,7 & 2,7\end{array}\right]$} & \\
\hline \multirow[t]{5}{*}{ Thevetia } & 24 & 21,4 & {$\left[\begin{array}{ll}25,5 & 18,5\end{array}\right]$} & \multirow{5}{*}{$<0,001$} \\
\hline & 48 & 15,5 & {$\left[\begin{array}{ll}15,5 & 11,3\end{array}\right]$} & \\
\hline & 72 & 9,8 & {$\left[\begin{array}{lll}8,5 & 6,2\end{array}\right]$} & \\
\hline & 96 & 4,7 & {$\left[\begin{array}{lll}4,5 & 3,3\end{array}\right]$} & \\
\hline & 120 & 3,1 & {$\left[\begin{array}{ll}3,7 & 2,7\end{array}\right]$} & \\
\hline
\end{tabular}

Tableau 9 : Modèle d'estimation de la valeur B résultant de la régression Cox pour les produits utilisés y inclus les coefficients de Wald.

\begin{tabular}{|c|c|c|c|c|c|c|}
\hline Produits & $\begin{array}{c}\text { Stade } \\
\text { Plutella }\end{array}$ & B & SE & Wald & ddl & Prob. \\
\hline Lambda & L3 & 6,286 & 0,922 & 46,474 & 1 & 0,000 \\
\hline Topbio & L3 & 5,891 & 0,901 & 40,417 & 1 & 0,000 \\
\hline Tephrosia & L3 & 5,339 & 0,865 & 39,894 & 1 & 0,000 \\
\hline CNSL à & L3 & 5,280 & 0,838 & 35,249 & 1 & 0,000 \\
\hline & L3 & 4,841 & 0,814 & 32,653 & 1 & 0,000 \\
\hline Thevetia & L3 & 4,802 & 0,801 & 30,216 & 1 & 0,000 \\
\hline
\end{tabular}

Tableau 10 : Modèle d’estimation de la valeur B résultant de la régression Cox pour les produits utilisés y inclus les coefficients de Wald.

\begin{tabular}{ccccccc}
\hline Produits & Stade Plutella & B & SE & Wald & ddl & Prob. \\
\hline Lambda & L2 & 68,287 & 4,713 & 209,897 & 1 & 0,000 \\
Topbio & L2 & 14,254 & 1,057 & 181,989 & 1 & 0,000 \\
\hline
\end{tabular}




\begin{tabular}{lcccccc}
\hline Tephrosia & L2 & 13,585 & 1,037 & 171,701 & 1 & 0,000 \\
CNSL & L2 & 12,496 & 0,983 & 161,438 & 1 & 0,000 \\
Ricinus & L2 & 11,934 & 0,952 & 157,217 & 1 & 0,000 \\
Thevetia & L2 & 11,113 & 0,895 & 154,308 & 1 & 0,000 \\
\hline \multicolumn{4}{l}{ B = Valeur B de la régression Cox ; SE = Erreur Standard ; Wald = coefficient de Wald ; ddl } \\
= degré de liberté ; Prob. = Probabilité
\end{tabular}

\section{Discussion}

D’une manière générale, les résultats obtenus des différentes expérimentations au laboratoire montrent que les pesticides botaniques à base de $T$. purpurea, $R$. communis, $T$. neriifolia et du baume de cajou extraction à froid utilisés sont toxiques aux larves de $P$. xylostella. Ces résultats corroborent les études sur l'effet larvicides de différents extraits à base de plantes par (Kétho et al., 2002; Sanda et al., 2006; Agboka et al., 2009). Le taux de mortalité des larves a augmenté dans le temps pour atteindre des pics en fonction des doses. Les différents résultats obtenus montrent également que la mortalité des larves de $P$. xylostella est fonction de la dose qu'elles reçoivent. Il ressort des résultats que les mortalités moyennes cumulées augmentent avec les concentrations des différents pesticides. L'étude des effets des pesticides botaniques sur le développement et le comportement alimentaire de $P$. xylostella a montré que tous les traitements ont eu un impact significatif sur la mortalité et l'alimentation des larves de P. xylostella. Le taux de mortalité des larves a augmenté dans le temps pour atteindre des pics en fonction des doses. L'effet larvicide des extraits de $R$. communis avait déjà été mis en exergue par (Olsnes, 2004; Kumar et al., 2007; Lui et al., 2007, Tounou A.K. et al., 2011, sur P. xylostella, par Aouinty et al. (2006) sur quatre espèces de moustiques, Culex pipiens (L.), Aedes caspius (Pallas), Culiseta longiareolata (Aitken) et Anopheles maculipennis (Meigen) Anani et al. (2004) et par Laghdaf et Ferji, (2005 ) sur les nématodes. La toxicité de la plante est due à la présence de ricine, une substance soluble dans l'eau, glycoprotéine concentrée dans la graine endosperme. T. neriifolia est une plante à intérêt médicinal à cause de sa propriété toxique (Garima et Amla, (2011). L’activité insecticide des extraits a été prouvée par plusieurs auteurs dans le domaine agricole. En effet, les extraits des organes de la plante ont montré une activité biocide sur C. maculatus (Mollah et Islam, 2007) et contre les charançons adultes de maïs (Wanyika et al., 2009) et sur les moustiques Anopheles gambiae (AKPO et al 2017). Les travaux réalisés par Chougourou et al., (2012) ont prouvé aussi l'efficacité biocide de l'huile extraite des amandes des graines sur les larves de Musca domestica. Des études similaires 
réalisées en Tanzanie ont montré aussi l'effet biocide des extraits de $T$. purpurea sur les larves de moustiques (Puyvelde et al., 2010). L'effet larvicide du CNSL a été prouvé par AKPO et al (2017) sur A. gambiae et par Azonkpin et al 2019 sur $H$. armigera. Le taux de mortalités a été progressif pour tous les produits en fonction de la dose, mais néanmoins, il existe une différence significative entre les différents produits testés sur les larves $(\mathrm{P}<0,001)$. Au stade L2 et L3, le taux de mortalité des larves à une dose d'application de $10 \%$ des différents pesticides botaniques utilisés et les témoins de références sont statistiquement différent. Ce taux de mortalité a été nettement supérieur sur les larves L2, lorsque ces mêmes doses ont été appliquées sur les stades L3 ; ceci est dû probablement à la taille plus élevée des larves L3, comparativement à celle des L2. Ces résultats sont similaires à ceux obtenus par Bateman et al. (1996) qui ont trouvé que l'infection des criquets par les champignons est fonction de leur poids. Au-delà de 3\% de l'huile de R. communis, et de $T$. neriifolia ; la réponse effet/dose n'est plus perceptible sur les larves L2, en raison probablement de la grande variabilité dans la mortalité d’une répétition à l'autre alors qu'il faut aller jusqu'à $1 \% T$. purpurea et de CNSL avant que le taux de mortalité soit proche des témoins négatifs. L'émergence des adultes a également été en fonction à la fois du pesticide botanique utilisé et des doses testées. Les quatre pesticides botaniques et les témoins de références utilisés ont eu un effet néfaste sur la chrysalidation et par suite sur l'émergence des adultes de $P$. xylostella. On note une baisse sensible de l'émergence des adultes qui est également dépendante des doses utilisées. Ce constat a été précédemment fait sur $P$. xylostella chez qui les extraits de feuilles de neem ont régulé la croissance des larves en entrainant une diminution du taux d'adultes émergés (Mondedji, 2010). Il ressort que les doses de 1 et 3\% des pesticides botaniques ont enregistré le plus fort taux de chrysalides et l'émergences des adultes. Les chrysalides issues des plus fortes doses des pesticides botaniques et les témoins de références ont une émergence assez faible comparés au plus faibles doses et au témoin négatif qui a enregistrés respectivement $96,4 \%$ et $95,12 \%$ du taux de chrysalide et l'émergences des adultes. Les doses d'application de 100\%, 75\%,50\%,25\% et $10 \%$ des pesticides botaniques ont enregistré $100 \%$ de mortalité pendant 9 jours sur les larves L2. Ces résultats confirment l'idée (Thomas et al., 1997), vu que l'insecte déjà infecté n'arrive plus à s'alimenter correctement et meurt. D'une manière générale, à l'émergence, des femelles sont plus nombreuses que les males. Ce résultat est contraire à celui obtenu par Douro (2011) suite au contrôle des larves de 3ème et de 4ème stade de $H$. armigera par les champignons entomopathogènes Beauveria bassiana et Metarhizium anisopliae par contre ce taux élevé de femelles émergées est similaire à celui observé par Surekha Devi (2009) suite à l'exposition de larves de H. armigera à une souche de Bacillus thuringiensis. En effet, la présence des pesticides 
botaniques a réduit significativement la consommation des feuilles par les larves. Les surfaces de feuilles de chou traitées aux différents extraits d'huile végétales consommées par les larves de $P$. xylostella furent globalement plus faibles que sur les feuilles témoin. Plus la concentration est élevée, plus la feuille est inappétence pour les larves. Dans une étude effectuée par Anam et al. (2006) une réduction significative des surfaces de feuilles de niébé traitées avec différentes concentrations d'huile de neem $(0,25 \% ; 0,5 \% ; 1 \% ; 2 \%$; et 4\%) consommé par les larves de Epilachna dodecastigma Wied. a été constatée en comparaison au témoin. Suite à un traitement de 24h ; 28,880 $\mathrm{mm} 2$ de feuilles ont été consommées contre 40,101 mm2 pour le témoin. Les effets antiappétants observés sont en fait fortement corrélés avec les réponses sensorielles des chémorécepteurs sur les pièces buccales des insectes (Mordue (Luntz) et al. 2000). De la position de ces récepteurs (tarses, pièces buccales, cavité buccale) dépend le comportement alimentaire de l'insecte. Mais outre la dissuasion alimentaire suite à l'inappétence des feuilles traitées, la mortalité et la chrysalidation des larves sont liées à des perturbations des mues. Les effets sur la mue sont dus à des perturbations dans la synthèse et la libération des ecdystéroïdes (hormones de la mue) par inhibition de la libération de l'hormone provenant des glandes prothoraciques (Mordue (Luntz) et Nisbet, 2000). Chez des insectes ayant reçu par injection de l'azadirachtine avant la production d'hormone, on a noté le blocage total de l'ecdysteroïde (Mordue (Luntz) et Nisbet, 2000). Cela a pour conséquence l'inhibition du développement de l'insecte puis de la mue larvaire et a entrainé la mort.

En ce qui concerne l'efficacité dans le temps des pesticides utilisés pour le contrôle des larves de $P$. xylostella, quelle que soit la durée d'exposition, les DL50 obtenues sont plus faibles respectivement pour le produit chimique de synthèse Lambda cyalothrine, Topbio, T. purpurea, CNSL, $R$. communis et T. neriifolia. Au début des tests, les pesticides ont eu une action répulsive sur $P$. xylostella. En effet, les larves ont quitté les feuilles traitées avec les pesticides. En dehors de cette action répulsive, l'étude des effets de ces pesticides ont montré chez $P$. xylostella des impacts sur le développement, la mue et la reproduction. En comparaison au témoin, les taux de mortalités élevés ont été relevés au niveau des populations de plutella nourris sur des feuilles traitées avec Lambda cyalothrine et les pesticides botaniques. La dose de $1 \%$ des pesticides a augmenté, la mortalité chez P. xylostella en comparaison au témoin. Des études similaires avaient été précédemment fait sur les pucerons par Lowery et al. (1993), qui ont montré qu'une dose de 1\% d'huile de neem a permis une réduction significative de la population du puceron en comparaison au contrôle. Les pesticides botaniques testés ont régulé la croissance de $P$. xylostella. En effet, une perturbation de la mue chez l'insecte a été observée chez des individus nourris sur des feuilles traitées. Cette perturbation chez les larves semble être due au blocage de la sécrétion 
des hormones de la mue. Ce blocage entraine l'arrêt du développement morphogénétique de la larve qui de ce fait, ne peut pas atteindre le stade adulte. Les coefficients de régression positifs pour les deux pesticides indépendamment des ravageurs prédisent une augmentation de la mortalité des ravageurs dans le temps. Quel que soit le pesticide chimique de synthèse ou botanique, la comparaison des DL50 temporelles obtenues montre que la quantité de pesticides nécessaire à l'élimination les larves du stade 2 est inférieure à celle requise pour éliminer les larves du troisième stade $P$. xylostella. La DL50 de T. purpurea nécessaire pour contrôler la moitié des larves du troisième stade 48 heures après traitement est de 13,4\% d'huile de T. purpurea contre $8,1 \%$ pour contrôler les larves du stade 2 . Cette forte différence pourrait être due au poids de chaque insecte. En effet, de façon globale, la dose létale s'exprime généralement en unité de substance par unité de masse. Les larves de 3ème stade de $P$. plutella étant plus grosse que le deuxième stade, il faudrait alors plus de substance pour les éliminer. Ce constat a été fait par Bateman et al. (1996) qui ont trouvé que l'infection des criquets par les champignons entomopathogènes est fonction de leur poids. De même, les travaux de Douro (2011) ont révélé qu'il faut une dose de Metarhizium anisopliae plus élevée pour contrôler les larves de 4ème stade de H. armigera par rapport aux larves de 3ème stade. A ce facteur lié à la masse pourrait également s'ajouter le mode d'alimentation de l'insecte.

\section{Conclusion}

En conclusion nous pouvons retenir que les différentes huiles végétales et le baume de cajou extraction à froid testés ont régulé la croissance de la population de $P$. xylostella utilisées en augmentant la mortalité des larves réduisant ainsi le taux d'adultes émergés. Les résultats obtenus après les tests biologiques réalisés ont révélé l'effet dose-réponse entre les larves traitées et les doses des pesticides utilisées. De même, le temps d'exposition influence la dose de pesticide à utiliser. Pour chaque pesticide, aussi bien les concentrations et le temps d'exposition ont montré des effets significatifs utiles pour la gestion et le développement de $P$. xylostella. Une analyse comparative des pesticides botaniques a montré une efficacité plus élevée respectivement de Topbio, de l'huile de Tephrosia, du CNSL à froid, de l'huile de Ricinus et de Thevetia .En perspective nous envisageons la caractérisation chimique des différentes huiles végétales utilisées et proposé une formulation en vue de déterminer la dose d'application optimale de ces pesticides botaniques en milieu réel.

\section{Remerciements}

Nous remercions l'Agence Universitaire de la Francophonie (AUF), pour leur aide financière à travers le Projet de Recherche Partenarial 2018. 
Nous remercions également l'entreprise Biophyto SARL pour sa contribution au projet.

\section{References:}

1. Abbott, W.S., 1925. A method of computing the effectiveness of an insecticide. Journal of Economic Entomology 18 (2): 265-267.

2. Agboka K, Agbodzavu K M, Tamò M, Vidal S, 2009. Effects of plant extracts and oil emulsions on the maize cob borer Mussidia nigrivenella (Lepidoptera: Pyralidae) in laboratory and field experiments. International Journal of Tropical Insect Science 29: 185194

3. Akpo A., 2017. Evaluation de l'efficacité des extraits des Plantes locales pour le contrôle des vecteurs du paludisme résistant aux pyréthrinoïdes au Bénin (Afrique de l'Ouest). Thèse de Doctorat de l'Université d'Abomey-Calavi. 253p

4. Anam M., Ahmad M. \& Haque M.A. (2006). Efficacy of Neem Oil on the Biology and Food Consumption of Epilachna Beetle, Epilachna dodecastigma (Wied.). J Agric Rural Dev 4(1\&2), 83-88 http://www.cabdirect.org/search.html?q=au\%3A\%22Shobha+.

5. Anani, B.Y., Gounou S., Chabi-Olaye A., Smith H. \& Schulthess F. (2004). The effect of neem (Azadirachta indica A. Juss) oil on oviposition, development and reproductive potentials of Sesamia calamistis Hampson (Lepidoptera: Noctuidae) and Eldana saccharina Walker (Lepidoptera: Pyralidae). The Royal Entomological Society, Agricult. Forest Entomol. 6: 223-232.

6. Aouinty B, Oufara S, Mellouki F, Mahari S, 2006. Evaluation préliminaire de l’activité larvicide des extraits aqueux des feuilles du ricin (Ricinus communis L.) et du bois de thuya (Tetraclinis articulata (Vahl) Mast.) sur les larves de quatre moustiques culicidés: Culex pipiens (Linné), Aedes caspius (Pallas),Culiseta longiareolata (Aitken) et Anopheles maculipennis (Meigen). Biotechnology,Agronomy, Society and Environment 10: 67

7. Argenti O. 2010. Approvisionnement et distribution des aliments dans les villes. Symposium international: Horticulture urbaine et périurbaine au siècle des villes. Symposium international Dakar, République du Sénégal, 6-9 décembre 2010 :30

8. AZONKPIN. S., Chougourou.D., Bokonon. A, Dossou. J.\& Ahonton .L., 2019 : Efficacite Du Baume De Cajou Contre Les Chenilles Carpophages Du Cotonnier Au Nord Du Benin European Scientific Journal Vol.14, No.24 ISSN: 1857 - 7881

9. Bateman R., Carey M., Batt D., Prior C., Abraham Y., Moora D., Jenkins N. \& Fenlon, J. (1996). Screening for virulent isolates of 
Entomopathogenic fungi against the Desert locust, Shistocerca gregaria (ForskAl). Biocont. Sc. and Technol 6: $549-560$.

10. Chougourou C. D., Dellouh P. L., Agbaka A., N’guessan K. R., Gbenou J. D., 2012. Toxicité et effets répulsifs de certaines huiles extraites des plantes locales béninoises sur la mouche domestique Musca domestica L. (Diptera Muscidae). Journal of Applied Biosciences 55 : 3953- 3961.

11. CHILCUTT, C.F. \& TABASHNIK, B.E. 1997. Host-mediated competition between the pathogen Bacillus thuringiensis and the parasitoid Cotesia plutellae of the diamondback moth (Lep.: Plutellidae). Environmental Entomology. 26: 38-45.

12. Douro Kpindou O. K. (2011). Potrntialités des entomopathogènes Metarhizium anisopliae (Hypocreales: Clavicipitaceae) et Beauveria bassiana (Hypocreales: Ophiocordycipitaceae) dans la gestion de Helicoverpa armigera (Hûbner) (Lepidoptera: Noctuidae) au Bénin. Thèse de doctorat Univ. de Lomé Togo, 186 p.

13. FAOSTAT. 2013. (http:// faostat.org). Food and Agriculture Organisation, United Nation.

14. FURLONG, M.J., WRIGHT, J. \& DOSDALL, L.M. 2012. Diamondback Moth Ecology and Management: Problems, Progress and Prospects. Annuel Review Entomology 58: 517-41.

15. Garima Z., Amla B., 2011. Thevetia peruviana (Pers.) Schum: A plant with enormous therapeutic potential. Journal of Pharmacy Research 2011,4(12), 4461-4464.

16. Glitho I.A., Ketoh G.K., Nuto P.Y., Amevoin S.K. \& Huignard J. (2008). Approches non toxiques et non polluantes pour le contrôle des populations d'insectes nuisibles en Afrique du Centre et de l'Ouest. In Biopesticides d’origine végétale (2è édition), Regnault $\mathrm{R}$, Philogène BJR, Vincent C (eds). Editeur Tec et Doc / Lavoisier : 207-217.

17. Guilloux T. 2000. Etude de la variabilité biologique, biochimique et génétique de populations d’origines géographiques différentes de Cotesia plutellae (Kurdjumov) (Hymenoptera Braconidae), parasitoide de la teigne des Brassicacées Plutella xylostella (L.) (Lepidoptera : Yponomeutidae). Thèse de Doctorat d'université, Université Paul

18. Gupta PD, Thorsteinson AJ. 1998. Food plant relationship of diamondback moth (Plutella 171 maculipennis Curt.) II. Sensory relationship ofd ovoposition of the adult female. Entomol. Exp. Appl 3: 305-314. Université Paul Valery, Montpellier III. 215p.

19. Hill TA, Foster RE. 2000. Effect of insecticides on the diamondback moth (Lepidoptera: Plutellidae) and its parasitoid Diadegma insulare (Hymenoptera: Ichneumonidae). J. Econ. 
a. Entomol., 93 (3) : 763-768

20. HOOKS, C.R.R. \& JOHNSON, M.W. 2003. Impact of agricultural diversification on the insect community of cruciferous crops. Crop Protection. 22: 223-238.

21. HUANG, Z., ALI, S., REN, S.X. \& WU, J.H. 2010. Effect of Isaria fumosoroseus on mortality and fecundity of Bemisia tabaci and Plutella xylostella. Insect Science and its Application 17: 140-148.

22. James, B.; C. Atcha; I. Godonou et H. Baimey (2005). Summary of activities and achievements, 2003-2005, Healthy vegetables through participatory IPM in peri-urban areas of Benin, International Institute of Tropical Agriculture (IITA), pp 49

23. James B, Atcha -Ahowe C, Godonou I, Baimey H, Goergen G, Sikirou R, Toko M. 2010. Gestion intégrée des nuisibles en production maraîchère : Guide pour les agents de vulgarisation en Afrique de l'Ouest. Institut international d'agriculture tropicale

a. (IITA), Ibadan, Nigeria. p120.

24. Kétoh GK, Ghlitho AI, Huignard J, 2002. Susceptibility of the bruchid Callosobruchus maculatus (Coleoptera: Pteromalidae) and its parasitoid Dinarmus basalis (Hymenoptera: Petromalidae) to three essential oils. Journal of Economic Entomology 95: 174-182.

25. Kumar O, Lakshmana Rao PV, Pradhan S, Jayaraj R, Bhaskar AS, Nashikkar AB, Vijayaraghavan R, 2007. Dose dependent effect of ricin on DNA damage and antioxidant enzymes in mice. Cell and Molecular Biology (Noisy.-le-grand) 53: 92-102.

26. Macharia I, Löhr B, De Groote H. 2005. Assessing the potential impact of biological control of Plutella xylostella (diamondback moth) in cabbage production in Kenya. Crop Prot., 24 981-989.

27. MONDEDJI, A.D. 2010. Potentiel d'utilisation d'extraits de feuilles de Neem (Azadirachta indica A. Juss) et de papayer (Carica papaya L.) dans le contrôle des insectes ravageurs du chou (Brassica oleracea L.) en zones urbaines et périurbaines au sud du Togo. Thèse de doctorat, Université de Lomé, Togo, p195.

28. Mollah J.U. \& Islam W., 2007: Toxicity of Thevetia peruviana (Pers) Schum. Extract to Adults of Callosobruchus maculatus F. (Coleoptera: Bruchidae). Agric Rural Dev 5(1 \& 2), 105-109

29. Mordue (Luntz) A. J. et Nisbet A. J., 2000. Azadirachtin from the neem tree Azadirachta indica: its action against insects An. Soc. Entomol. Bras. vol.29 no.4, Londrina Dec. 2000.

30. Mordue (Luntz), A.J., Simmonds M.S.J., Ley S.V., Blaney W.M., Mordue W., Nasiruddin M. \& Nisbet A.J. (1998). Actions of azadirachtin, a plant allelochemical, against insects. Pestic. Sci. 54: 277-284. 
31. Muhammad O, Tsukuda R, Oki Y, Fujisaki K, Nakasuji F. 1994. Influences of wild crucifers on life history traits and flight ability of the diamondback moth, Plutella xylostella (Lep.: Yponomeutidae). Researches in Population ecology, 36: 53-62.

32. Maddox J.V., 1982. Use of insect pathogens in pest management. In: Metcalf R.L. \& Luckmann W.H., eds. Introduction to insect pest management. 2nd ed. New York, USA: Wiley, 175-216

33. Laghdaf, T., Ferji, Z. 2005 : Evalution de quleques plantes nématicides et produits. Nationle sur les Substences Naturelles : La valorisation et dévloppement, Errachidia, Marco. P 29-30.

34. LIU, S.S., WANG, X.G., GUO, S.J., HE, J.H., \& SONG, H.M. (1995). A survey of insect parasitoids of Plutella xylostella and the seasonal abundance of the major parasitoids in Hangzhou, China. In: Sivapragasan, A., Loke, W.H., Hussan, A.K. \& Lim G.S. (Eds.) The management of Diamondback Moth and other Crucifer Pests. In Proceedings of the Third International Workshop (pp. 61-66). Mardi, Kuala Lumpur, Malaysia

35. LIU, S.S., WANG, X.G., GUO, S.J., HE, J.H. \& SHI, Z. 2007. Seasonal abundance of the parasitoid complex associated with the diamondback moth, Plutella xylostella (Lepidoptera: Plutellidae) in Hangzhou, China. Bulletin of Entomological Research 90:221-231.

36. Lowery D.T., Isman M.B.\& Bhard, N.L. (1993). Laboratory and field evaluation of neem for the control of aphids (Homoptera:Aphididae). J. Econ. Entomol., 6: 864-870.

37. Olsnes, S., Refsnes, K., Pihl, A. (2004): Mechanism of action of the toxic lectins abrin and ricin. Nat. 249:627-631

38. Puyvelde LV, De Kimpe N., Mudaheranwa J.P., Gasiga A., Schamp N. et al., 2010: Journal of Natural Products. 50, 349 p.

39. Sanda K, Koba K, Poutouli W, Idrissou N, Agbossou AB, 2006. Pesticidal propertties of Cymbopogon schoenatus against the Diamondback moth Plutella xylostella L (Lepidoptera: Hyponomeutidae). Discovery and Innovation 19: 220-225.

40. Sarfraz, M., Keddie, A.B., Dosdall, L.M., 2005. Biological control of the diamondback moth, Plutella xylostella: A review Published online 20 July 2005. Biocontrol Sci. Techn. 15, 763-789.

41. Shelton AM, Roush RT, Wang P, Zhao JZ. 2007. Resistance to insect pathogens and strategies to manage resistance: An update. In: Field Manual of Techniques in Invertebrate Pathology. Kluwer Academic Press, p. 793-811.

42. Slotkin T.A., Stadler A., Skavicus S. \& Seidler F.J., 2016. Adolescents and adults differ in the immediate and long-term impact of nicotine 
administration and withdrawal on cardiac norepinephrine. Brain Res. Bull., 122, 71-75.

43. Tabashnik BF, Mau RFL. 1990. Suppression of Diamondback moth (Lepidoptera: Plutellidae) oviposition by overhead irrigation. J. Econ. Entomol., 79 : 189-191.

44. Thomas M.B., Blanford S. \& Lomer C.J., 1997. Reduction of feeding by the variegated grasshopper, Zonocerus variegatus, following infection by the fungal pathogen, Metarhizium flavoviride. Biocontrol Sci. Technol., 7, 327-334.

45. Tounou A. K., Mawussi G., Amadou S., Agboka K., Gumedzoe Y. M. D., Sanda K., 2011. Bio-insecticidal effects of plant extracts and oil emulsions of Ricinus communis L. (Malpighiales: Euphorbiaceae) on the diamondback, Plutella xylostella L. (Lepidoptera: Plutellidae) under laboratory and semi-field conditions. J. Appl. Biosci., 43, 28992914.

46. Verkerk RHJ, Wright DJ. 1996. Multitrophic interactions and management of the diamondback moth: a review. Bulletin Entomological Research 86: 205-216.

47. Wainwright H., Wanyamay C. \& Cherotich N., 2013. Biopesticides and their commercialisation in Africa. In: Proceedings of the First International Conference on Pesticidal Plants, 21-24 January 2013, Egerton University and ICIPE, Nairobi, Kenya, 189-191.

48. Wanyika H.N., Kareru P.G., Keriko J.M., Gachanja A.N, Kenji G.M. \& Mukiira N.J, 2009: Contact toxicity of some fixed plant oils and stabilized natural pyrethrum extracts against adult maize weevils (Sitophilus zeamais Motschulsky). African Journal of Pharmacy and Pharmacology Vol. 3 (2), 066-069

49. WARWICK, S.I., FRANCIS, A. \& MULLIGAN, G.A. 2003. Brassicaceae of Canada. Government of Canada. Available: http://www.cbif.gc.ca/spp_pages/brass/index_e.php. Biocontrol Sci. Technol Pp 401-425. 